\title{
Temperature-dependent nonlinear analysis of shallow shells: A theoretical approach
}

\author{
P. Khazaeinejad*, A.S. Usmani \\ School of Engineering, The University of Edinburgh, The King's Buildings, Edinburgh EH9 \\ $3 J L, U K$
}

\begin{abstract}
The paper presents a theoretical formulation for the computation of temperaturedependent nonlinear response of shallow shells with single and double curvatures subjected to transverse mechanical loads while being exposed to through-depth non-uniform heating regimes such as those resulting from a fire. The material nonlinearity arises from taking into consideration the degradation of the material elastic behaviour at elevated temperatures under quasi-static conditions. Two types of boundary conditions are considered, both of which constrain the transverse deflections and allow the rotations about the edge axis to be free. One of the boundary conditions permits lateral translation (laterally unrestrained) and the other one does not (laterally restrained). A number of examples are solved for shallow shells under different types of loading conditions including: an exponential "short hot" fire leading to a high temperature over a relatively short duration; and an exponential "long cool" fire of lower temperature over a longer duration. The limits of the shallow shell equations are investigated through comparison studies. Results show that while current numerical approaches for analysis of laterally restrained shallow shells are often computationally intensive, the proposed approach offers an adequate level of accuracy with a rapid convergence rate for such structures.
\end{abstract}

Keywords: Geometric nonlinearity, Material nonlinearity,

\footnotetext{
${ }^{*}$ Corresponding author. Tel.: +44 (0) 1316505806.

Email address: p.khazaeinejad@ed.ac.uk (P. Khazaeinejad)
}

The final version of this paper has been published in Composite Structures, Volume 141, Pages 1-13. 
Temperature-dependent material properties, Composite shallow shell, Non-uniform heating, Structures in fire

\section{Introduction}

Shallow shells are widely used in the design of lightweight thin-walled structures. They can deliver useful load-carrying capacity by virtue of their curvatures, thereby effectively resisting the externally applied loads with optimum use of materials. The most common applications are in buildings (typically as roof structures), aerospace vehicles, ship hulls, pressure vessels, and car bodies. Most of these structures are potentially at risk of being exposed to severe nonuniform thermal gradients while also externally loaded, such as those resulting from a fire. The consequences of such loading conditions may lead to a reduction of the strength and stiffness of the structure and the deyelopment of large deflections, leading to the failure of the structure under the most severe cases. Research in this area has been often focused towards developing efficient shell finite elements (e.g. see Refs. [1 7]). Numerical techniques have been widely used to study the nonlinear behaviour of shells, mainly for their flexibility and ability in dealing with shell problems in comparison with theoretical approaches.

Kumar and Palaninathan 8 employed an eight-node degenerated layered shell element to investigate the geometric nonlinear response of laminated composite cylindrical panels to axial compression and central concentrated load. Their numerical results showed that boundary conditions (BCs) have significant influence on the load-carrying capacity of cylindrical panels so that those with free curved edges and hinged longitudinal edges undergo either limit point or bifurcation failure at very low load levels in comparison with other straight and clamped edge conditions. Using the differential quadrature method, Wang [9] solved the geometric nonlinear buckling problem of thin doubly-curved orthotropic shallow shells with hinged edges. The buckling loads obtained by this method were lower than semi-analytical solutions obtained by either adjacent equilibrium method or partitioned solution method. It was concluded that to 
ensure the convergence of the solutions, an alternative method should be used as using small load increment increases the computational time required for solving nonlinear problems.

Panda and Singh [10] developed a nonlinear finite element (FE) model to analyse the thermal buckling and post-buckling strength of laminated composite shallow cylindrical/hyperboloid shell panels subjected to uniform temperature rise. In their model, the full nonlinearity effect in the geometry was taken into account in a Green-Lagrange sense based on the higher order shear deformation theory. Their numerical results indicated that the post-buckling strength in thermal environment is higher for hyperboloid shell panels in eomparison with cylindrical panels. Altekin and Yukseler [11] employed finite difference and Newton-Raphson methods to solve the geometrically nonlinear axisymmetric bending problem of homogeneous and isotropic shallow spherical shells with either clamped or simply supported edges under axisymmetric loads. Their study showed that for partially loaded shells, the influence of the BCs on the central deflection of the shell is negligible. Civalek [12] used discrete singular convolution and differential quadrature methods for the nonlinear static and dynamic analysis of simply supported (laterally unrestrained) and clamped shallow spherical shells resting on elastic foundations. The dynamic analysis of shell structures are well documented in the literature (e.g., see Refs. [13 20]).

Alongside with the advances in numerical techniques, a significant improvement has also been observed in the capability of analytical and semi-analytical approaches to deal with many difficulties in shell nonlinear problems. Development of appropriate theoretical approaches is not only useful for benchmarking FE codes developed for shell-type structures but also for visualising internal structures in composite shells in order to develop much deeper insights into their load-carrying mechanisms. Woo and Meguid [21] studied the nonlinear analysis of simply supported (laterally unrestrained) shallow spherical shells with functionally graded (FG) material properties subjected to transverse mechanical loads and through-depth thermal gradients. The governing equations were established based on the von Kármán theory for large out-of-plane deflec- 
tions and were solved using series solutions. It was revealed that considering thermo-mechanical coupling effects in the shell formulation can affect the nonlinear response of the shell. Based on the Donnell thin shell theory, van Campen et al. 22] developed semi-analytical methods using the adjacent equilibrium method and partitioned solution method to study the stability behaviour of doubly-curved shallow orthotropic panels under external pressure. In the former method, solutions at the neutral equilibrium position were perturbated to calculate the bifurcation buckling load. However, in the latter method, solutions of the equilibrium and compatibility equations of the panel were perturbated to calculate both the bifurcation buckling and post-buckling solutions that are not confined in the initial post-buckling region. Comparing buckling loads for panels with simply supported and hinged BCs, they showed that the lower buckling load is produced for cases with hinged BCs due to pre-buckling compression.

The nonlinear analysis of an imperfect shallow spherical shell on a Pasternak foundation subjected to uniform loads was presented by Nie 23. The shell was assumed elastically restrained against rotational, out-of-plane and in-plane displacements. The asymptotic iteration method was applied to obtain an analytical expression for the external load and the central deflection of the shell. Numerical results indicated that imperfections cause a drop in the load-bearing capacity of the shell. Heuer and Ziegler [24] studied the thermal snap-through and snap-buckling of symmetrically layered shallow shells with polygonal planforms and laterally restrained BCs using a two degrees of freedom model derived from a Ritz-Galerkin approximation. Duc and Van Tung [25] studied the nonlinear response) of FG cylindrical panels to uniform lateral pressure and uniform and through-depth thermal gradients by an analytical approach associated with a Galerkin method. Formulation was based on the classical shell theory, considering the von Kármán-Donnell type of kinematic nonlinearity and initial geometrical imperfection. Three BCs were considered in their analysis arising from restriction on the lateral movement of curved and straight edges of the cylindrical panel. Temperature-dependency of material properties of the panel were not taken into consideration. Numerical results revealed that in-plane re- 
straint and temperature conditions play major roles in dictating the response of FG cylindrical panels.

Shen and his colleagues studied the post-buckling behaviour of FG cylindrical shells in thermal environments under an axial compression [26, 27], a lateral pressure [28], and a uniform temperature rise over the shell surface and through the shell thickness [29] using the classical shell theory with the von KármánDonnell-type of kinematic nonlinearity assumptions. Material properties of the shell were assumed to be temperature-dependent and graded in the thickness direction according to a simple power law distribution. A boundary layer theory of shell buckling, which includes the effects of nonlinear pre-buckling deformations, large deflections in the post-buckling range, and initial geometric imperfections of the shell was used. A singular perturbation technique was employed to obtain the buckling loads and post-buckling equilibrium paths. Yang et al. [30] evaluated the effects of thermal loads, temperature-dependent properties, initial geometric imperfection, volume fraction index, and geometrical parameters on the post-buckling behaviour of FG cylindrical panels with either simply supported (laterally unrestrained) or clamped edges subjected to a combined initial axial force and a uniform temperature change. Their analysis was based on the classical shell theory and the von Kármán-Donnell-type kinematic relations. The critical buckling temperature and the post-buckling temperature-deflection curves were determined using a semi-analytical differential quadrature-Galerkin method associated with an iterative algorithm. They reported that both the buckling temperature and the equilibrium path in the post-buckling regime become lower when the temperature-dependent properties are taken into account in the analysis.

Shahsiah et al. 31] obtained analytical solutions for the thermal instability of FG thin shallow spherical shells based on the Donnell-Mushtari-Vlasov theory. The shell was assumed under three types of thermal loading including a uniform temperature rise, a linear radial temperature, and a nonlinear radial temperature. Girish and Ramachandra [32] presented analytical solutions of the post-buckling problem of symmetric and antisymmetric cross-ply laminated 
cylindrical shell panels under thermo-mechanical loading based on higher order shear deformation theory. They reported that the shear deformation is less effective on the critical buckling loads of antisymmetric cross-ply shell panels in comparison to symmetric cross-ply shell panels. Nie et al. 33] studied the nonlinear buckling of imperfect orthotropic shallow shells on an elastic foundation using the asymptotic iteration method. Amabili 34 presented the large amplitude of the response of simply supported (laterally not fully unrestrained) doubly-curved shallow shells with rectangular planform to static and dynamic loads. He used the Donnell and Novozhilov shell theories retaining in-plane inertia to obtain the geometrically nonlinear response of the shell. Hamed et al. [35] theoretically and experimentally examined the failure behaviour of thin-walled shallow concrete domes. Their theoretical study included the development of an analytical model for the nonlinear behaviour of materials under failure levels of load, the creep and shrinkage of the concrete material, and the buckling of the dome.

An analytical approach was employed by Bich and Van Tung [36] to study the nonlinear stability of perfect and imperfect EG shallow spherical shells under uniform external pressure with and without considering the effects of uniform and through-depth thermal gradients. In their approach, one term approximation of deflection was used to determine the extremum buckling loads and load-deflection curves for laterally restrained and unrestrained shallow spherical shells. Material properties were assumed to be temperature-independent. Their results showed that while the effect of temperature field on the nonlinear response of FG shallow spherical shells under thermo-mechanical loads is significant, the effect of initial imperfection on the nonlinear response of mechanicallyloaded FG shallow spherical shells is weak. The nonlinear buckling behaviour of homogeneous and non-homogeneous orthotropic thin-walled truncated conical shells under axial load was presented by Sofiyev and Kuruoglu [37. The stability and compatibility equations of the problem were derived using the large deformation theory with the von Kármán-Donnell type of kinematic nonlinearity. It was reported that for long truncated conical shells, the effect of non-homogeneity 
on the nonlinear axial buckling load is negligible. Xue et al. 38] extended the von Kármán-Donnell shell theory for long cylindrical shells undergoing large flexural deflections. It was shown that the initial curvature has a significant influence on the behavior of long cylindrical shells. Recently, Tornabene et al. [39] studied the static deformations of doubly-curved and free-form sandwich shells with either the face sheets or the core made of FG materials. Their focus was on recovering accurate values of through-the-depth transverse normal and shear strains and stresses. The generalized differential quadrature method was employed to solve the system of governing equations for different higher order shear deformation theories. They found that stresses of FG structures are inbetween those for homogeneous structures made of the two constituents of the FG material.

In most composite shell analyses, spatially varying material properties are not employed and the analysis has been often limited to temperature sensitive material properties. Hence, in the present paper, the formulation by Khazaeinejad et al. 40 is extended to account for the temperature-dependent nonlinear response of shallow shells with single and double curvatures on a rectangular planform. A shallow shell is subjected to a transverse mechanical load while it is exposed to through-depth non-uniform temperature profiles. Assuming a comparatively small rise for the shell, its geometry is expressed using the Cartesian coordinates instead of curvilinear coordinates. Two limiting cases are considered for the shell BCs, assuming the shell edges to be either unrestrained or restrained against lateral translations while transverse deflections along the shell boundaries are restrained and rotations parallel to the shell boundary are free. Results are presented for three different cases, namely isotropic, orthotropic FG, and shallow shells with temperature-dependent material properties for both the chosen BCs. In latter case, the shell nonlinear response is studied under two common types of quasi-static fire exposure conditions including: a "short hot" exponential fire of high temperature over a short post-flashover duration [41]; and a "long cool" exponential fire with lower maximum temperature over a longer post-flashover period [41. Quiver plots of principal stresses and 


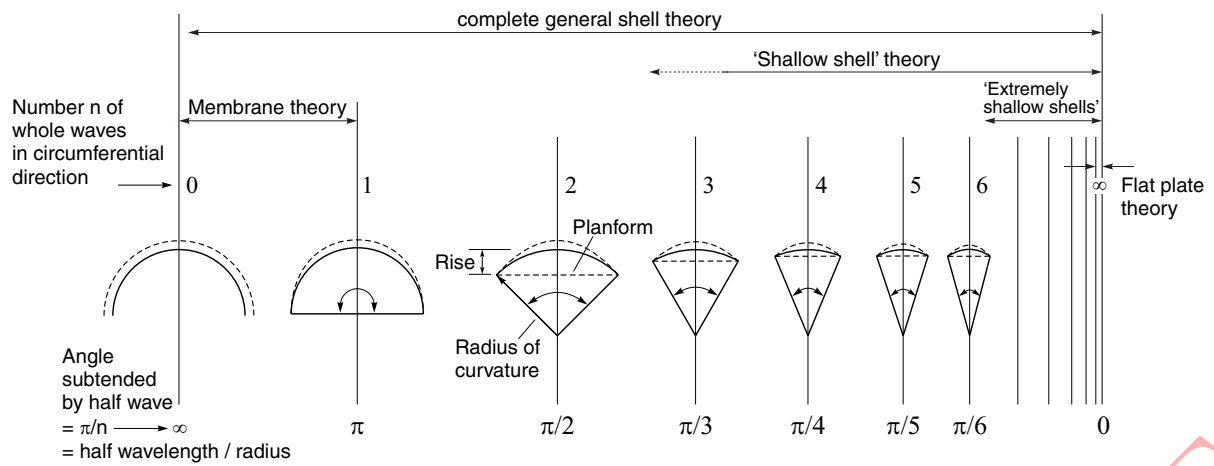

Figure 1: Hierarchy of various shell theories as a function of their applicable ranges of subtended angle. The figure is adapted from Donnell [42. A shallow shell typically has a rise of less than one-fifth of the smallest dimension of its planform. In the case of 'extremely shallow shells', the minimum radius of curvature of the shell is more than two times larger than its maximum planform dimension 43 .

membrane tractions are also graphically illustrated for the examples considered. Comparison studies between the results obtained from the proposed formulation and those obtained from FE analyses show that the proposed approach offers a rapid convergence rate as well as an adequate level of accuracy for 'extremely shallow shells' (see Figure 1).

\section{Kinematic and constitutive relations}

Consider a shallow shell with a rectangular planform in the Cartesian coordinates system $(x, y, z)$. The rise of the shell is assumed to be relatively small in comparison to its other dimensions. This is based on the shallow shell theory assumption in which the rise of a shallow shell above its planform is typically assumed to be less than one-fifth the smallest dimension of the planform (see Figure 11. The shell is assumed to undergo large deflections. In such a case, the von Kármán large deflection assumption which accounts for the stretching of the middle surface of the shell can be used. The shallow shell kinematic relations 
including thermal effects are

$$
\begin{aligned}
\varepsilon & =\left\{\begin{array}{c}
\frac{\partial u}{\partial x}+\frac{1}{2}\left(\frac{\partial w}{\partial x}\right)^{2}+\frac{w}{R_{x}} \\
\frac{\partial v}{\partial y}+\frac{1}{2}\left(\frac{\partial w}{\partial y}\right)^{2}+\frac{w}{R_{y}} \\
\frac{\partial u}{\partial y}+\frac{\partial v}{\partial x}+\frac{\partial w}{\partial x} \frac{\partial w}{\partial y}+\frac{2 w}{R_{x y}}
\end{array}\right\}+z\left\{\begin{array}{c}
-\frac{\partial^{2} w}{\partial x^{2}} \\
-\frac{\partial^{2} w}{\partial y^{2}} \\
-2 \frac{\partial^{2} w}{\partial x \partial y}
\end{array}\right\}+\left\{\begin{array}{c}
\alpha_{x x}(z, \theta) \theta(z) \\
\alpha_{y y}(z, \theta) \theta(z) \\
\alpha_{x y}(z, \theta) \theta(z)
\end{array}\right\} \\
& =\varepsilon_{0}+z \varepsilon_{1}+\varepsilon_{\theta}
\end{aligned}
$$

where $u, v$ and $w$ are the displacements of the middle surface of the shell in the $x, y$ and $z$-directions, respectively, $\alpha_{x x}(\theta, z), \alpha_{y y}(\theta, z)$, and $\alpha_{x y}(\theta, z)$ are the temperature-dependent transformed coefficients of thermal expansion, $\theta(z)$ represents the temperature increase through the depth of the shell, $R_{x y}$ represents the twist radius of the middle surface of the shell, and $R_{x}$ and $R_{y}$ are the radii of curvature of the undeformed shell, as illustrated in Figure 2 for shallow shells with single or double curvature.

The in-plane stresses vary linearly from the middle surface of the shell as follows

$$
\boldsymbol{\sigma}=\left\{\begin{array}{c}
\sigma_{x x} \\
\sigma_{y y} \\
\sigma_{x y}
\end{array}\right\}=\mathbf{Q}\left[\varepsilon_{0}+z \varepsilon_{1}+\varepsilon_{\theta}\right]
$$

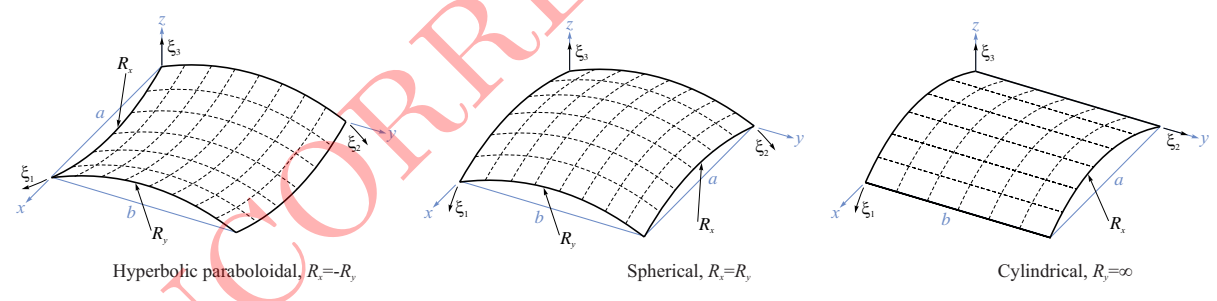

Figure 2: Common types of curvature for shallow shells with a rectangular planform. The curvilinear coordinates on the middle surface of the shells are replaced with a Cartesian coordinate system due to a comparatively small rise above the shells' rectangular planform. It must be noted that for untwisted shallow shells the radius $R_{x y}$ is infinite. 
The membrane tractions (forces) for the shallow shell can be obtained by integrating the in-plane stresses of the shell over its depth

$$
\mathbf{N}=\left\{\begin{array}{c}
N_{x x} \\
N_{y y} \\
N_{x y}
\end{array}\right\}=\mathbf{A} \varepsilon_{0}+\mathbf{B} \varepsilon_{1}-\mathbf{N}^{\theta}
$$

The moment resultants can also be obtained by twice integrating the in-plane stresses over the depth of the shell as follows

$$
\mathbf{M}=\left\{\begin{array}{l}
M_{x x} \\
M_{y y} \\
M_{x y}
\end{array}\right\}=\mathbf{B} \varepsilon_{0}+\mathbf{D} \varepsilon_{1}-\mathbf{M}^{\theta}
$$

where the coefficient matrices $\mathbf{A}, \mathbf{B}$ and $\mathbf{D}$ are related to the stiffness matrix $\mathbf{Q}$ by the following relations

$$
\left\{\begin{array}{l}
\mathbf{A} \\
\mathbf{B} \\
\mathbf{D}
\end{array}\right\}=\int_{-\frac{h}{2}}^{\frac{h}{2}} \mathbf{Q}\left\{\begin{array}{c}
1 \\
z \\
z^{2}
\end{array}\right\} d z
$$

In the above expressions, $\mathbf{N}^{\theta}$ and $\mathbf{M}^{\theta}$ are thermal stress and moment resultants, respectively, defined by

$$
\begin{aligned}
& \mathbf{N}^{\theta}=\int_{-\frac{h}{2}}^{\frac{h}{2}} \mathbf{Q}\left\{\begin{array}{l}
\alpha_{x x}(z, \theta) \\
\alpha_{y y}(z, \theta) \\
\alpha_{x y}(z, \theta)
\end{array}\right\} \theta(z) d z \\
& \mathbf{M}^{\theta}=\int_{-\frac{h}{2}}^{\frac{h}{2}} \mathbf{Q}\left\{\begin{array}{c}
\alpha_{x x}(z, \theta) \\
\alpha_{y y}(z, \theta) \\
\alpha_{x y}(z, \theta)
\end{array}\right\} \theta(z) z d z
\end{aligned}
$$

in which $\mathbf{Q}$ is the stiffness matrix associated with the material properties.

\section{Derivation of governing equations}

Under quasi-static conditions, the nonlinear response of shallow shells can be determined by solving a system of two coupled nonlinear equations simultaneously. This includes the compatibility and equilibrium equations of the shell. 
The compatibility equation relates the internal membrane forces caused by large deformations to the out-of-plane displacement of the shell. It can be obtained by taking the second derivatives of the strains and combining the resulting expressions as follows

$\frac{\partial^{2} \varepsilon_{x x}}{\partial y^{2}}+\frac{\partial^{2} \varepsilon_{y y}}{\partial x^{2}}-\frac{\partial^{2} \gamma_{x y}}{\partial x \partial y}=\left(\frac{\partial^{2} w}{\partial x \partial y}\right)^{2}-\frac{\partial^{2} w}{\partial x^{2}} \frac{\partial^{2} w}{\partial y^{2}}+\frac{1}{R_{y}} \frac{\partial^{2} w}{\partial x^{2}}+\frac{1}{R_{x}} \frac{\partial^{2} w}{\partial y^{2}}-\frac{2}{R_{x y}} \frac{\partial^{2} w}{\partial x \partial y}$

Since the membrane tractions vary only over the shell surface and not through the depth of the shell, they can be expressed by stress function $F(x, y)$ as follows

$$
\mathbf{N}=\left\{\begin{array}{c}
\frac{\partial^{2} F}{\partial y^{2}} \\
\frac{\partial^{2} F}{\partial x^{2}} \\
-\frac{\partial^{2} F}{\partial x \partial y}
\end{array}\right\}
$$

By rearranging Eq. (3) along with using Eq. (8), the middle plane strains can be written as

$$
\varepsilon_{0}=\mathbf{a N}+\mathbf{b} \varepsilon_{1}+\mathbf{a} \mathbf{N}^{\theta}
$$

where $\mathbf{a}$ and $\mathbf{b}$ are related to the stiffness matrices $\mathbf{A}$ and $\mathbf{B}$ as defined in the following

$$
\begin{aligned}
& \mathbf{a}=\mathbf{A}^{-1} \\
& \mathbf{b}=\mathbf{A}^{-1} \mathbf{B}
\end{aligned}
$$

As can be seen, the effect of material nonlinearity is reflected in the matrices a and b. Substituting Eqs. (8) and (9) into the strain compatibility equation (7) the following nonlinear equation is derived

$$
\begin{aligned}
& a_{22} \frac{\partial^{4} F}{\partial x^{4}}-2 a_{26} \frac{\partial^{4} F}{\partial x^{3} \partial y}+\left(2 a_{12}+a_{66}\right) \frac{\partial^{4} F}{\partial x^{2} y^{2}}-2 a_{16} \frac{\partial^{4} F}{\partial x \partial y^{3}}+a_{11} \frac{\partial^{4} F}{\partial y^{4}} \\
& -\left(\frac{\partial^{2} w}{\partial x \partial y}\right)^{2}+\frac{\partial^{2} w}{\partial x^{2}} \frac{\partial^{2} w}{\partial y^{2}}+b_{12} \frac{\partial^{4} w}{\partial x^{4}}+\left(2 b_{26}-b_{16}\right) \frac{\partial^{4} w}{\partial x^{3} \partial y} \\
& +\left(b_{11}+b_{22}-2 b_{66}\right) \frac{\partial^{4} w}{\partial x^{2} \partial y^{2}}+\left(2 b_{16}-b_{26}\right) \frac{\partial^{4} w}{\partial x \partial y^{3}}+b_{12} \frac{\partial^{4} w}{\partial y^{4}}-\frac{1}{R_{x}} \frac{\partial^{2} w}{\partial y^{2}} \\
& -\frac{1}{R_{y}} \frac{\partial^{2} w}{\partial x^{2}}+2 \frac{1}{R_{x y}} \frac{\partial^{2} w}{\partial x \partial y}=-a_{12} \frac{\partial^{2} N_{x x}^{\theta}}{\partial x^{2}}+a_{16} \frac{\partial^{2} N_{x x}^{\theta}}{\partial x \partial y}-a_{11} \frac{\partial^{2} N_{x x}^{\theta}}{\partial y^{2}}-a_{22} \frac{\partial^{2} N_{y y}^{\theta}}{\partial x^{2}} \\
& \quad+a_{26} \frac{\partial^{2} N_{y y}^{\theta}}{\partial x \partial y}-a_{12} \frac{\partial^{2} N_{y y}^{\theta}}{\partial y^{2}}-a_{26} \frac{\partial^{2} N_{x y}^{\theta}}{\partial x^{2}}+a_{66} \frac{\partial^{2} N_{x y}^{\theta}}{\partial x \partial y}-a_{16} \frac{\partial^{2} N_{x y}^{\theta}}{\partial y^{2}}
\end{aligned}
$$


In the above equation, $F$ and $w$ are both unknown. Therefore, another equation is required, that is the equilibrium equation of the shell which relates the axial forces to the out-of-plane displacement of the shell as follows

$$
\begin{gathered}
\frac{\partial^{2} M_{x x}}{\partial x^{2}}+2 \frac{\partial^{2} M_{x y}}{\partial x \partial y}+\frac{\partial^{2} M_{y y}}{\partial y^{2}}+N_{x x} \frac{\partial^{2} w}{\partial x^{2}}+N_{y y} \frac{\partial^{2} w}{\partial y^{2}} \\
+2 N_{x y} \frac{\partial^{2} w}{\partial x \partial y}-\left(\frac{N_{x x}}{R_{x}}+\frac{N_{y y}}{R_{y}}+\frac{2 N_{x y}}{R_{x y}}\right)+q=0
\end{gathered}
$$

where $q(x, y)$ represents the transverse mechanical load. From substituting Eq. (9) into Eq. (4), the moment resultants can be rewritten as

$$
\mathbf{M}=\mathbf{c N}+\mathbf{d} \varepsilon_{1}+\mathbf{c} \mathbf{N}^{\theta}-\mathbf{M}^{\theta}
$$

where the coefficient $\mathbf{c}$ and $\mathbf{d}$ are related to the stiffness matrices $\mathbf{A}, \mathbf{B}$, and $\mathbf{D}$ via

$$
\begin{aligned}
\mathbf{c} & =\mathbf{B A}^{-1} \\
\mathbf{d} & =\mathbf{D}-\mathbf{B A}^{-1} \mathbf{B}
\end{aligned}
$$

Upon substitution of Eq. (13) into Eq. (12), the nonlinear equilibrium equation can be obtained as

$$
\begin{gathered}
d_{11} \frac{\partial^{4} w}{\partial x^{4}}+2\left(d_{16}+d_{16}\right) \frac{\partial^{4} w}{\partial x^{3} \partial y}+2\left(d_{12}+2 d_{66}\right) \frac{\partial^{4} w}{\partial x^{2} y^{2}}+4 d_{26} \frac{\partial^{4} w}{\partial x \partial y^{3}}+d_{22} \frac{\partial^{4} w}{\partial y^{4}} \\
-\frac{\partial^{2} F}{\partial y^{2}}\left(\frac{\partial^{2} w}{\partial x^{2}}-\frac{1}{R_{x}}\right)+2 \frac{\partial^{2} F}{\partial x \partial y}\left(\frac{\partial^{2} w}{\partial x \partial y}-\frac{1}{R_{x y}}\right)-\frac{\partial^{2} F}{\partial x^{2}}\left(\frac{\partial^{2} w}{\partial y^{2}}-\frac{1}{R_{y}}\right)-c_{12} \frac{\partial^{4} F}{\partial x^{4}} \\
-\left(2 c_{26}-c_{16}\right) \frac{\partial^{4} F}{\partial x^{3} \partial y}-\left(c_{11}+c_{22}-2 c_{66}\right) \frac{\partial^{4} F}{\partial x^{2} y^{2}}-\left(2 c_{16}-c_{26}\right) \frac{\partial^{4} F}{\partial x \partial y^{3}}-c_{12} \frac{\partial^{4} F}{\partial y^{4}}=q \\
+c_{11} \frac{\partial^{2} N_{x x}^{\theta}}{\partial x^{2}}+c_{12} \frac{\partial^{2} N_{x x}^{\theta}}{\partial y^{2}}+2 c_{16} \frac{\partial^{2} N_{x x}^{\theta}}{\partial x \partial y}+c_{12} \frac{\partial^{2} N_{y y}^{\theta}}{\partial x^{2}}+c_{22} \frac{\partial^{2} N_{y y}^{\theta}}{\partial y^{2}}+2 c_{26} \frac{\partial^{2} N_{y y}^{\theta}}{\partial x \partial y} \\
+c_{16} \frac{\partial^{2} N_{x y}^{\theta}}{\partial x^{2}}+c_{26} \frac{\partial^{2} N_{x y}^{\theta}}{\partial y^{2}}+2 c_{66} \frac{\partial^{2} N_{x y}^{\theta}}{\partial x \partial y}-\frac{\partial^{2} M_{x x}^{\theta}}{\partial x^{2}}-\frac{\partial^{2} M_{y y}^{\theta}}{\partial y^{2}}-2 \frac{\partial^{2} M_{x y}^{\theta}}{\partial x \partial y}
\end{gathered}
$$

The most common way of solving the differential equations (11) and (15) is using trigonometric functions, depending on the BCs specified for the shell.

\section{Solution of governing equations}

In this study, two types of BCs are assumed: (1) edges laterally unrestrained, thereby permitting lateral translations, while out-of-plane translations are restrained along the shell edges and rotations about the edge axes are free. This 
type of $\mathrm{BC}$ is referred to as the 'laterally unrestrained' BC; and (2) edges laterally restrained, in which all translations are restricted along the shell edges while the rotation about the edge axes remains free. This type of $\mathrm{BC}$ is referred to as the 'laterally restrained' BC.

The assumed BCs are reasonable limiting cases bracketing the conditions that may be found in real shell structures, except for cases where rotational restraints are important. The following expression satisfies the BCs for the out-of-plane displacement

$$
w(x, y)=\sum_{m=1}^{\infty} \sum_{n=1}^{\infty} w_{m n} S_{m n}
$$

where $S_{m n}=\sin (\bar{m} x) \sin (\bar{n} y)$ with $\bar{m}=m \pi / a$ and $\bar{n}=n \pi / b$. The thermal force and moment resultants can also be expressed by Fourier series as follows

$$
\left\{\begin{array}{c}
N_{x x}^{\theta}(x, y) \\
N_{y y}^{\theta}(x, y) \\
M_{x x}^{\theta}(x, y) \\
M_{y y}^{\theta}(x, y)
\end{array}\right\}=\sum_{m=1}^{\infty} \sum_{n=1}^{\infty}\left\{\begin{array}{c}
N_{m \eta x x}^{\theta} \\
N_{m n y y}^{\theta} \\
M_{m n x x}^{\theta} \\
M_{m n y y}^{\theta}
\end{array}\right\} S_{m n}
$$

where the coefficients are calculated by

$$
\left\{\begin{array}{l}
N_{m n x x}^{\theta} \\
N_{m n y y}^{\theta} \\
M_{m n x x}^{\theta} \\
M_{m n y y}^{\theta}
\end{array}\right\}=\frac{4\left[-1+(-1)^{m}\right]\left[-1+(-1)^{n}\right]}{m n \pi^{2}}\left\{\begin{array}{c}
N_{x x}^{\theta} \\
N_{y y}^{\theta} \\
M_{x x}^{\theta} \\
M_{y y}^{\theta}
\end{array}\right\}
$$

Different transverse mechanical loading conditions can be considered by expressing the quantity $q$ in a double Fourier series as follows

$$
q(x, y)=\sum_{m=1}^{\infty} \sum_{n=1}^{\infty} q_{m n} S_{m n}
$$

where

$$
q_{m n}=\frac{4}{a b} \int_{0}^{a} \int_{0}^{b} q(x, y) S_{m n} d y d x
$$

The following loading conditions are then derived: 
For a uniformly distributed load (UDL) of magnitude $q$ :

$$
q_{m n}=\frac{4\left[-1+(-1)^{m}\right]\left[-1+(-1)^{n}\right]}{m n \pi^{2}} q
$$

For a sinusoidal loading of magnitude $q$ where $m=n=1$ :

$$
q_{m n}=q
$$

For a point load of $q$ applied at coordinates $\left(x_{0}, y_{0}\right)$ :

$$
q_{m n}=\frac{4 q_{0}}{a b} \sin \frac{m \pi x_{0}}{a} \sin \frac{n \pi y_{0}}{b}
$$

For the stress function an expression satisfying the stress-free edges case (for the laterally unrestrained BC) and undeformed edges case (for the laterally restrained $\mathrm{BC}$ ) may then be taken as

$$
F(x, y)=\frac{P_{x} y^{2}}{2 b h}+\frac{P_{y} x^{2}}{2 a h}+\sum_{m=1}^{\infty} \sum_{n=1}^{\infty} F_{m n} S_{m n}
$$

where $P_{x}$ and $P_{y}$ are equivalent reaction loads at the shell boundaries. In the case of laterally restrained BC, such loads can be obtained using the following expressions for the elongation of the shell in the $x$ and $y$ directions

$$
\begin{array}{r}
\int_{0}^{a} \frac{\partial u}{\partial x} d x=\int_{0}^{a}\left(a_{11} \frac{\partial^{2} F}{\partial y^{2}}+a_{12} \frac{\partial^{2} F}{\partial x^{2}}+b_{11} \frac{\partial^{2} w}{\partial x^{2}}+b_{12} \frac{\partial^{2} w}{\partial y^{2}}+a_{11} N_{x x}^{\theta}\right. \\
\left.+a_{12} N_{y y}^{\theta}-\frac{1}{2}\left(\frac{\partial w}{\partial x}\right)^{2}\right) d x \\
\int_{0}^{b} \frac{\partial v}{\partial y} d y=\int_{0}^{b}\left(a_{12} \frac{\partial^{2} F}{\partial y^{2}}+a_{22} \frac{\partial^{2} F}{\partial x^{2}}+b_{12} \frac{\partial^{2} w}{\partial x^{2}}\right. \\
+b_{22} \frac{\partial^{2} w}{\partial y^{2}}+a_{12} N_{x x}^{\theta} \\
\left.+a_{22} N_{y y}^{\theta}-\frac{1}{2}\left(\frac{\partial w}{\partial y}\right)^{2}\right)^{(25 \mathrm{~b})} d y
\end{array}
$$

Setting the edge displacements to zero after substituting Eqs. (16) and 24 into Eqs. 25 and performing the integration, yields the following expressions for the reaction loads 


$$
\begin{aligned}
P_{x} & =\frac{b h\left(a_{22} \bar{m}^{2}-a_{12} \bar{n}^{2}\right)}{8\left(a_{11} a_{22}-a_{12}^{2}\right)} w_{m n}^{2}+\frac{\left[-1+(-1)^{m}\right]\left[-1+(-1)^{n}\right] b h \bar{n}^{2}}{m n \pi^{2}} F_{m n} \\
& +\frac{b h\left[-1+(-1)^{m}\right]\left[-1+(-1)^{n}\right]\left[\left(a_{22} b_{11}-a_{12} b_{12}\right) \bar{m}^{2}+\left(a_{22} b_{12}-a_{12} b_{22}\right) \bar{n}^{2}\right]}{m n \pi^{2}\left(a_{11} a_{22}-a_{12}^{2}\right)} w_{m n} \\
& +\frac{\left[-1+(-1)^{m}\right]\left[-1+(-1)^{n}\right] b h}{m n \pi^{2}\left(a_{11} a_{22}-a_{12}^{2}\right)}\left(\frac{a_{22}}{R_{x}}-\frac{a_{12}}{R_{y}}\right) w_{m n}-b h N_{x x}^{\theta} \\
P_{y} & =\frac{a h\left(a_{11} \bar{n}^{2}-a_{12} \bar{m}^{2}\right)}{8\left(a_{11} a_{22}-a_{12}^{2}\right)} w_{m n}^{2}+\frac{\left[-1+(-1)^{m}\right]\left[-1+(-1)^{n}\right] a h \bar{m}^{2}}{m n \pi^{2}} F_{m n} \\
& +\frac{a h\left[-1+(-1)^{m}\right]\left[-1+(-1)^{n}\right]\left[\left(a_{11} b_{12}-a_{12} b_{11}\right) \bar{m}^{2}+\left(a_{11} b_{22}-a_{12} b_{12}\right) \bar{n}^{2}\right]}{m n \pi^{2}\left(a_{11} a_{22}-a_{12}^{2}\right)} w_{m n} \\
& +\frac{\left[-1+(-1)^{m}\right]\left[-1+(-1)^{n}\right] a h}{m n \pi^{2}\left(a_{11} a_{22}-a_{12}^{2}\right)}\left(\frac{a_{11}}{R_{y}}-\frac{a_{12}}{R_{x}}\right) w_{m n}-a h N_{y y}^{\theta}
\end{aligned}
$$

By contrast, when the laterally unrestrained BC is imposed, such reaction loads are zero and the following expressions can then be obtained for the in-plane displacements $u$ and $v$

$$
\begin{aligned}
u=\sum_{m=1}^{\infty} \sum_{n=1}^{\infty}[ & -\frac{\bar{m}^{2}}{4} w_{m n}^{2}\left(x+\frac{\sin 2 \bar{m} x}{2 \bar{m}}\right) \sin ^{2} \bar{n} y \\
& +\frac{b_{11} \bar{m}^{2}+b_{12} \bar{n}^{2}}{\bar{m}} w_{m n} \cos \bar{m} x \sin \bar{n} y+\frac{w_{m n}}{\bar{m} R_{x}} \cos \bar{m} x \sin \bar{n} y \\
& \left.+\frac{a_{12} \bar{m}^{2}+a_{11} \bar{n}^{2}}{\bar{m}} F_{m n} \cos \bar{m} x \sin \bar{n} y+a a_{11} N_{x x}^{\theta}+a a_{12} N_{y y}^{\theta}\right] \\
v=\sum_{m=1}^{\infty} \sum_{n=1}^{\infty}[ & -\frac{\bar{n}^{2}}{4} w_{m n}^{2} \sin ^{2} \bar{m} x\left(y+\frac{\sin 2 \bar{n} y}{2 \bar{n}}\right) \\
& +\frac{b_{12} \bar{m}^{2}+b_{22} \bar{n}^{2}}{\bar{n}} w_{m n} \sin \bar{m} x \cos \bar{n} y+\frac{w_{m n}}{\bar{n} R_{y}} \sin \bar{m} x \cos \bar{n} y \\
& \left.+\frac{a_{22} \bar{m}^{2}+a_{12} \bar{n}^{2}}{\bar{n}} F_{m n} \sin \bar{m} x \cos \bar{n} y+b a_{12} N_{x x}^{\theta}+b a_{22} N_{y y}^{\theta}\right]
\end{aligned}
$$

In the case of a laminated shell, the quantities $N_{x y}^{\theta}, M_{x y}^{\theta}, Q_{16}, Q_{26}$ are zero. It is clear that the coefficients of the in-plane displacements are also temperature-dependent. Considering the expressions (16)-(24) into the governing equations (11) and (15), the compatibility and equilibrium equations are 
obtained in terms of $F_{m n}$ and $w_{m n}$

$$
\begin{aligned}
& {\left[a_{22} \bar{m}^{4}+\left(2 a_{12}+a_{66}\right) \bar{m}^{2} \bar{n}^{2}+a_{11} \bar{n}^{4}\right] F_{m n} S_{m n}} \\
& -\quad \bar{m}^{2} \bar{n}^{2} w_{m n}^{2}\left(C_{m n}^{2}-S_{m n}^{2}\right) \\
& +\left[\left(b_{11}+b_{22}-2 b_{66}\right) \bar{m}^{2} \bar{n}^{2}+b_{12} \bar{m}^{4}+b_{12} \bar{n}^{4}+\frac{\bar{n}^{2}}{R_{x}}+\frac{\bar{m}^{2}}{R_{y}}\right] w_{m n} S_{m n} \\
& -\left[\left(a_{12} N_{m n x x}^{\theta}+a_{22} N_{m n y y}^{\theta}\right) \bar{m}^{2}\right. \\
& \left.+\left(a_{11} N_{m n x x}^{\theta}+a_{12} N_{m n y y}^{\theta}\right) \bar{n}^{2}\right] S_{m n}=0 \\
& {\left[d_{11} \bar{m}^{4}+\left(2 d_{12}+4 d_{66}\right) \bar{m}^{2} \bar{n}^{2}+d_{22} \bar{n}^{4}\right] w_{m n} S_{m n}} \\
& \quad-\left[c_{12} \bar{m}^{4}+\left(c_{11}+c_{22}-2 c_{66}\right) \bar{m}^{2} \bar{n}^{2}+c_{12} \bar{n}^{4}+\frac{\bar{n}^{2}}{R_{x}}+\frac{\bar{m}^{2}}{R_{y}}\right] F_{m n} S_{m n} \\
& \quad+\left(\frac{P_{x} \bar{m}^{2}}{b h}+\frac{P_{y} \bar{n}^{2}}{a h}\right) w_{m n} S_{m n}-2 \bar{m}^{2} \bar{n}^{2} F_{m n} w_{m n}\left(S_{m n}^{2}-C_{m n}^{2}\right) \\
& \quad+\frac{P_{x}}{b h R_{x}}+\frac{P_{y}}{a h R_{y}}+\left[\left(c_{11} N_{m n x x}^{\theta}+c_{12} N_{m n y y}^{\theta}\right) \bar{m}^{2}\right. \\
& \left.\quad+\left(c_{12} N_{m n x x}^{\theta}+c_{22} N_{m n y y}^{\theta}\right) \bar{n}^{2}\right] S_{m n} \\
& \quad-\left(\bar{m}^{2} M_{m n x x}^{\theta}+\bar{n}^{2} M_{m n y y}^{\theta}\right) S_{m n}-q_{m n} S_{m n}=0
\end{aligned}
$$

where $C_{m n}=\cos (\bar{m} x) \cos (\bar{n} y)$. If the left part of Eqs. (28) and 29) are denoted by $X_{1}$ and $X_{2}$, respectively, then from the following equations

$$
\int_{0}^{a} \int_{0}^{b}\left\{\begin{array}{l}
X_{1} \\
X_{2}
\end{array}\right\} S_{m n}=0
$$

two algebraic equations can be derived as follows

$$
\begin{aligned}
F_{m n}= & \frac{1}{\left(a_{22} \bar{m}^{4}+\left(2 a_{12}+a_{66}\right) \bar{m}^{2} \bar{n}^{2}+a_{11} \bar{n}^{4}\right)}\left[4 \bar{m}^{2} \bar{n}^{2} H_{m n} w_{m n}^{2}\right. \\
& +\left[b_{12} \bar{m}^{4}+\left(b_{11}+b_{22}-2 b_{66}\right) \bar{m}^{2} \bar{n}^{2}+b_{12} \bar{n}^{4}+\frac{\bar{n}^{2}}{R_{x}}+\frac{\bar{m}^{2}}{R_{y}}\right] w_{m n} \\
& \left.+\left[\left(a_{12} N_{m n x x}^{\theta}+a_{22} N_{m n y y}^{\theta}\right) \bar{m}^{2}+\left(a_{11} N_{m n x x}^{\theta}+a_{12} N_{m n y y}^{\theta}\right) \bar{n}^{2}\right]\right]
\end{aligned}
$$

where

$$
\begin{aligned}
& H_{m n} \\
& =\frac{-1+2(-1)^{m}+2(-1)^{n}-(-1)^{3 m}-(-1)^{3 n}-3(-1)^{m+n}+(-1)^{3 m+n}+(-1)^{m+3 n}}{3 m n \pi^{2}}
\end{aligned}
$$


Likewise, the following load-deflection equation is derived from Eq. 29

$$
\varsigma_{1} w_{m n}+\varsigma_{2} F_{m n}+\varsigma_{3} F_{m n} w_{m n}+\varsigma_{4}=0
$$

where the coefficients are defined by

$$
\begin{aligned}
\varsigma_{1}= & \frac{P_{x} \bar{m}^{2}}{b h}+\frac{P_{y} \bar{n}^{2}}{a h}+d_{11} \bar{m}^{4}+\left(2 d_{12}+4 d_{66}\right) \bar{m}^{2} \bar{n}^{2}+d_{22} \bar{n}^{4} \\
\varsigma_{2}= & -\left[c_{12} \bar{m}^{4}+\left(c_{11}+c_{22}-2 c_{66}\right) \bar{m}^{2} \bar{n}^{2}+c_{12} \bar{n}^{4}+\frac{\bar{n}^{2}}{R_{x}}+\frac{\bar{m}^{2}}{R_{y}}\right] \\
\varsigma_{3}= & 8 \bar{m}^{2} \bar{n}^{2} H_{m n} \\
\varsigma_{4}= & \frac{4\left[-1+(-1)^{m}\right]\left[-1+(-1)^{n}\right]}{m n \pi^{2}}\left(\frac{P_{x}}{b h R_{x}}+\frac{P_{y}}{a h R_{y}}\right) \\
& +\left(c_{11} N_{m n x x}^{\theta}+c_{12} N_{m n y y}^{\theta}\right) \bar{m}^{2}+\left(c_{12} N_{m n x x}^{\theta}+c_{22} N_{m n y y}^{\theta}\right) \bar{n}^{2} \\
& -\left(\bar{m}^{2} M_{m n x x}^{\theta}+\bar{n}^{2} M_{m n y y}^{\theta}\right)-q_{m n}
\end{aligned}
$$

Once the material and geometric parameters are known, the coefficient $w_{m n}$ can be readily obtained by substituting the stress function from Eq. (31) into Eq. (33). Having resolved the load-deflection equation, the out-of-plane displacement of the shallow shell can be accurately/calculated for an adequate number of series terms, depending on the specified support conditions.

\section{Results and discussion}

In this section, a number of examples are solved for isotropic, orthotropic FG, and temperature-dependent shallow shells with both the chosen BCs. For all these examples, representations of either principal stresses or membrane tractions in the shell are graphically illustrated to gain a deeper insight into the nonlinear response of shallow shells with single or double curvature. Table 1 lists the dimensionless quantities used in the following examples.

\subsection{Isotropic shallow shells}

To verify the accuracy of the proposed approach, a series of comparison studies are presented. First, four square isotropic shallow cylindrical shells with different curvatures which were modelled using the FE software ABAQUS 
Table 2: Comparison studies for a square isotropic shallow cylindrical shell with the laterally restrained $\mathrm{BC}$ subjected to a UDL using three terms in the series solutions

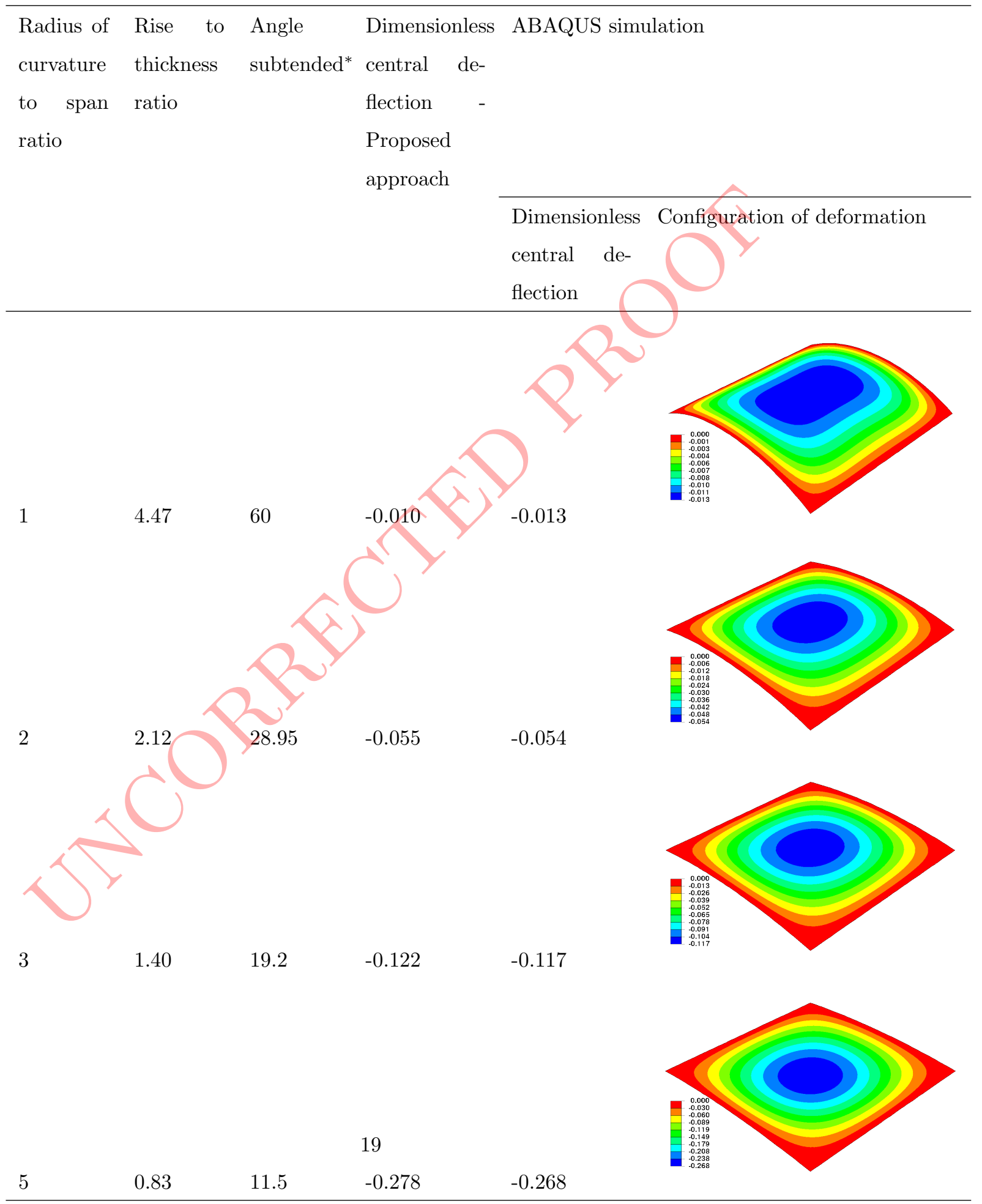

${ }^{*}$ See Figure 1 
Table 3: Comparison studies for a square isotropic shallow cylindrical shell with the laterally unrestrained $\mathrm{BC}$ subjected to a UDL using one term in the series solutions

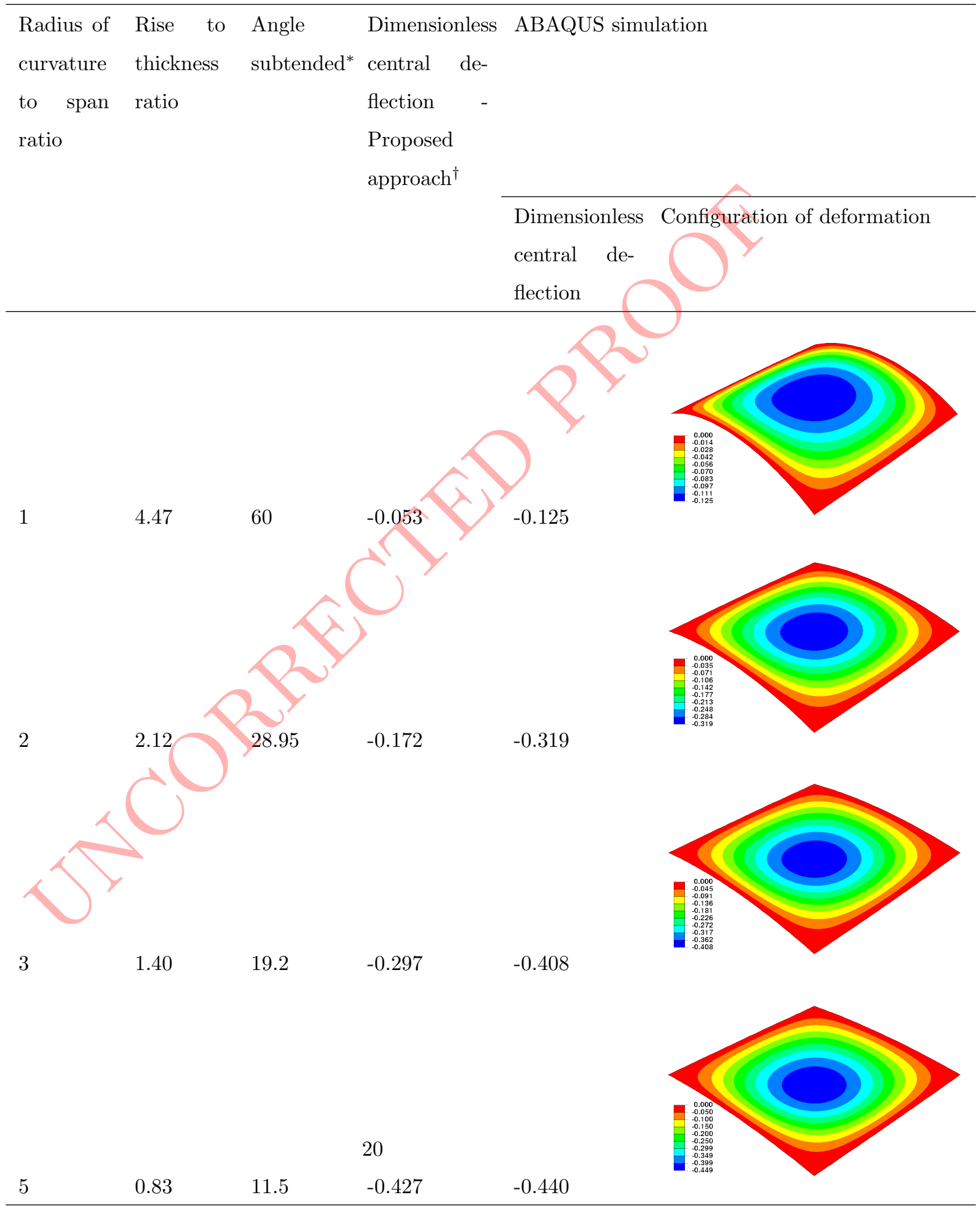

\footnotetext{
${ }^{*}$ See Figure 1
}

${ }^{\dagger}$ For this $\mathrm{BC}$, the present formulation is only accurate for extremely shallow shells (e.g. $R_{x} / a=5$ ). 


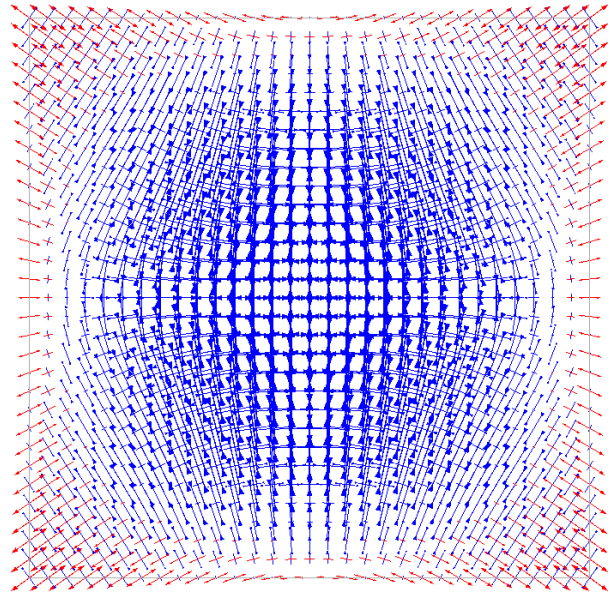

(a) Proposed approach - Top surface

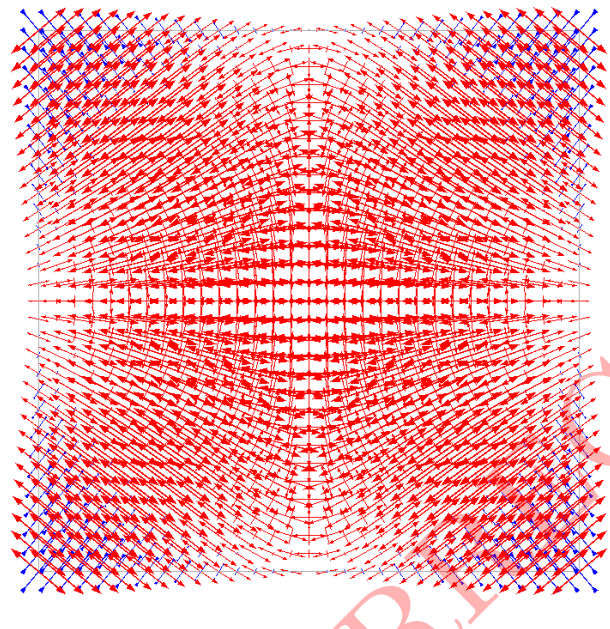

(c) Proposed approach - Bottom surface

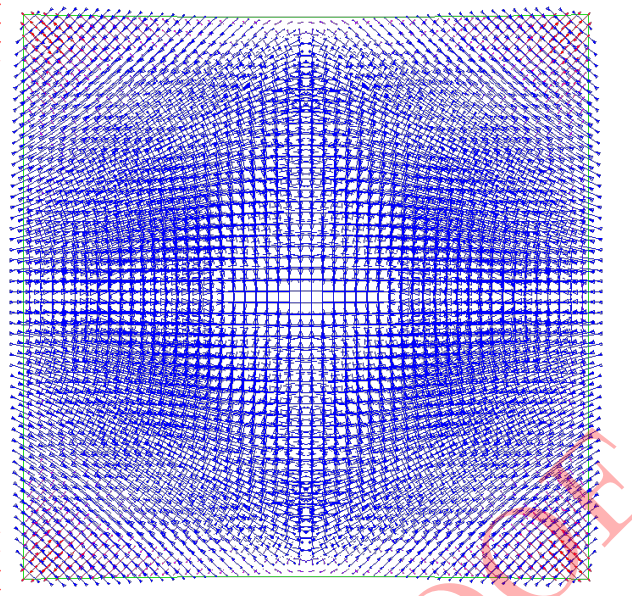

(b) FE analysis - Top surface

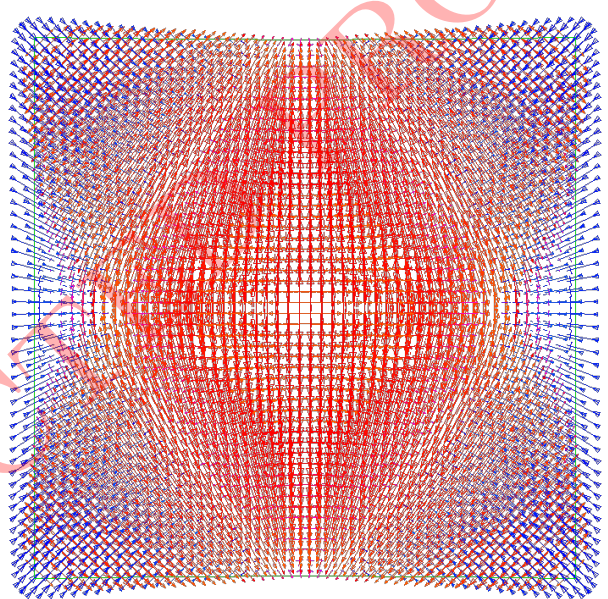

(d) FE analysis - Bottom surface

Figure 3: Quiver plots of principal stresses on the top and bottom surfaces of a square isotropic shallow cylindrical shell with the laterally restrained BC subjected to a UDL. Blue arrows represent compressive stresses and the red ones represent tensile stresses. Five series terms are used in the proposed formulation to produce the stress trajectories.

with respect to the unrestrained boundaries is related to the structure moving from larger to smaller curvature. The model seems to work significantly better for smaller curvatures than for larger curvatures.

For extremely shallow shells $\left(R_{x} / a=5\right)$ in Tables 2 and 3 , quiver plots of 


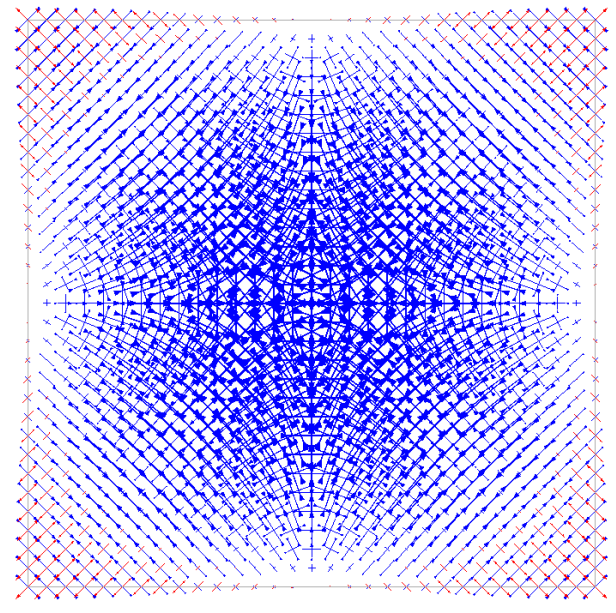

(a) Proposed approach - Top surface

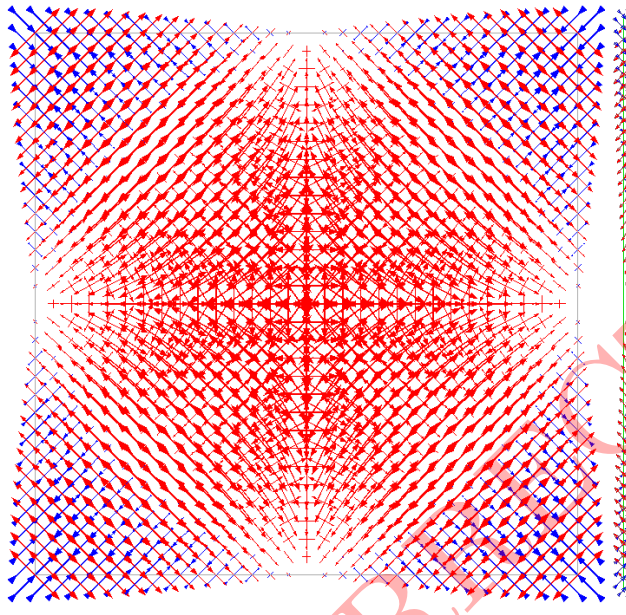

(c) Proposed approach - Bottom surface

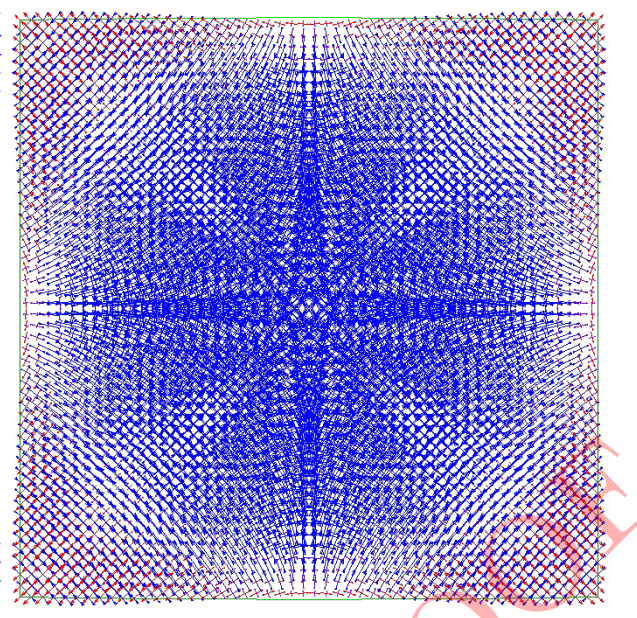

(b) FE analysis - Top surface

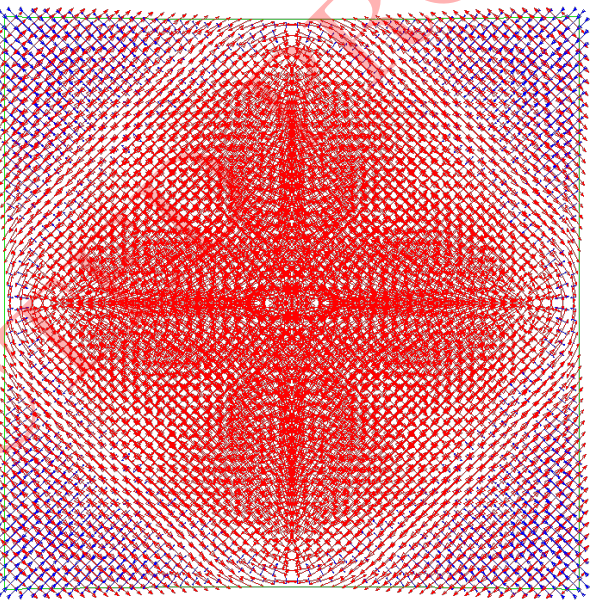

(d) FE analysis - Bottom surface

Figure 4: Quiver plots of principal stresses on the top and bottom surfaces of a square isotropic shallow cylindrical shell with the laterally unrestrained BC subjected to a UDL. Blue arrows represent compressive stresses and the red ones represent tensile stresses. Five series terms are used in the proposed formulation to produce the stress trajectories.

all principal stresses on the top and bottom surfaces of the shell are illustrated in Figures 3 and 4 using GiD postprocessing framework. These figures are obtained using the proposed approach and ABAQUS for square shallow cylindrical shells with the laterally restrained and unrestrained BCs. The lengths of the 
vectors are proportional to their magnitudes. Both Figures 3 and 4 show that the top surface of the shell under both BCs is predominantly under compression while predominantly tension occurs at the bottom surface of the shell, which suggests a behaviour not unlike plate bending. However, closer inspection shows that the stress trajectories and distribution patterns are quite different for the two BCs. The case of the unrestrained BC shows that the tensile stresses at the bottom surface are concentrated towards the centre with a large area of compression surrounding them, similar to a compressive ring surrounding laterally unrestrained plates under large deflections [40. It can also be seen from the figures that the general stress patterns predicted by the proposed approach match quite well with those predicted by the ABAQUS.

\subsection{FG shallow shells}

In this section, an FG shallow shell under large displacements is analysed. Good thermal resistance of FG materials at high temperatures has been widely accepted in the scientific community. A typical FG material is compositionally graded from a refractory ceramic to metal. Accordingly, the material properties of an FG shell vary continuously throughout the depth of the shell according to the volume fraction of constituents given by either a power-law distribution [44, 45] or an exponential distribution [46. The former distribution has been commonly used for modelling FG shells. For an orthotropic FG shell, the stiffness coefficients are given by

$$
\mathbf{Q}=\left(\begin{array}{ccc}
\frac{E 1(z)}{1-\nu_{12} \nu_{21}} & \frac{E 2(z) \nu_{12}}{1-\nu_{12} \nu_{21}} & 0 \\
& \frac{E 2(z)}{1-\nu_{12} \nu_{21}} & 0 \\
& & \frac{E 2(z)}{2\left(1+\nu_{12}\right)}
\end{array}\right)
$$

where E1 and E2 are elastic modulii referring to the axes $x$ and $y$ and are defined as

$$
\left\{\begin{array}{l}
E 1(z) \\
E 2(z)
\end{array}\right\}=\left\{\begin{array}{c}
E 1_{t}-E 1_{b} \\
E 2_{t}-E 2_{b}
\end{array}\right\}\left(\frac{1}{2}+\frac{z}{h}\right)^{\lambda}+\left\{\begin{array}{c}
E 1 b \\
E 2 b
\end{array}\right\}
$$


where $\lambda(\geq 0)$ is the volume fraction index and subscripts $E i_{t}$ and $E i_{b}$ represent the material properties of the top and bottom surfaces of layer $i$ in the shell, respectively.

Figure 5 a shows the results of dimensionless maximum deflection of a square orthotropic FG shallow spherical shell with the laterally unrestrained BC subjected to a UDL which are compared with the results obtained from a meshless local Petrov-Galerkin (MLPG) formulation 47]. The shell has thickness of $0.0127 \mathrm{~m}$, span of $0.254 \mathrm{~m}$, and radius of curvature to span ratio of 10 . It is subjected to a UDL of $2.07 \times 10^{6} \mathrm{~N} / \mathrm{m}^{2}$ directed upwards (i.e. bottom surface loading). A quadratic variation of the elastic moduli is considered $(\lambda=2)$ with the elastic modulus on the bottom surface being $E 1_{b}=0.6895 \times 10^{10} \mathrm{~N} / \mathrm{m}^{2}$ and $E 2_{b}=E 1_{b} / 2$ and the elastic modulus on the top surface being $E 2_{t}=$ $0.6895 \times 10^{10} \mathrm{~N} / \mathrm{m}^{2}$ and $E 1_{t}=2 E 2_{t}$. For this example, the deflection is presented in a different dimensionless form, namely deflection of shallow shell over deflection of its corresponding flat shell $\left(R_{x}=R_{y}=\infty\right)$. Figure 5 a shows that an acceptable level of accuracy can be achieved for extremely shallow spherical shells with the laterally unrestrained BC using the proposed approach. This is justified by the convergence of the solution in Figure $5 \mathrm{~b}$ which shows that the FE prediction is closer to the theoretical prediction obtained using three series terms in the calculations. It is also found that the maximum deflection value at the centre of an orthotropic FG shallow shell is about 1.13 times greater than the maximum deflection of its corresponding flat shell because of the low magnitude of elastic modulus chosen at the bottom surface of the shell.

Quiver plots of all dimensionless principal membrane tractions (forces) for the same orthotropic FG shallow shell with both the laterally restrained and unrestrained BCs are illustrated in Figure 6. As expected, a completely symmetric distribution is formed for both the BCs. Since an upwardly directed UDL is applied, the behaviour of the shell with the laterally restrained BC is completely dominated by tensile membrane forces. However, in the case of laterally unrestrained BC, compressive membrane stresses occur around the shell corners while tensile membrane forces are formed in the central zone of the shell. 

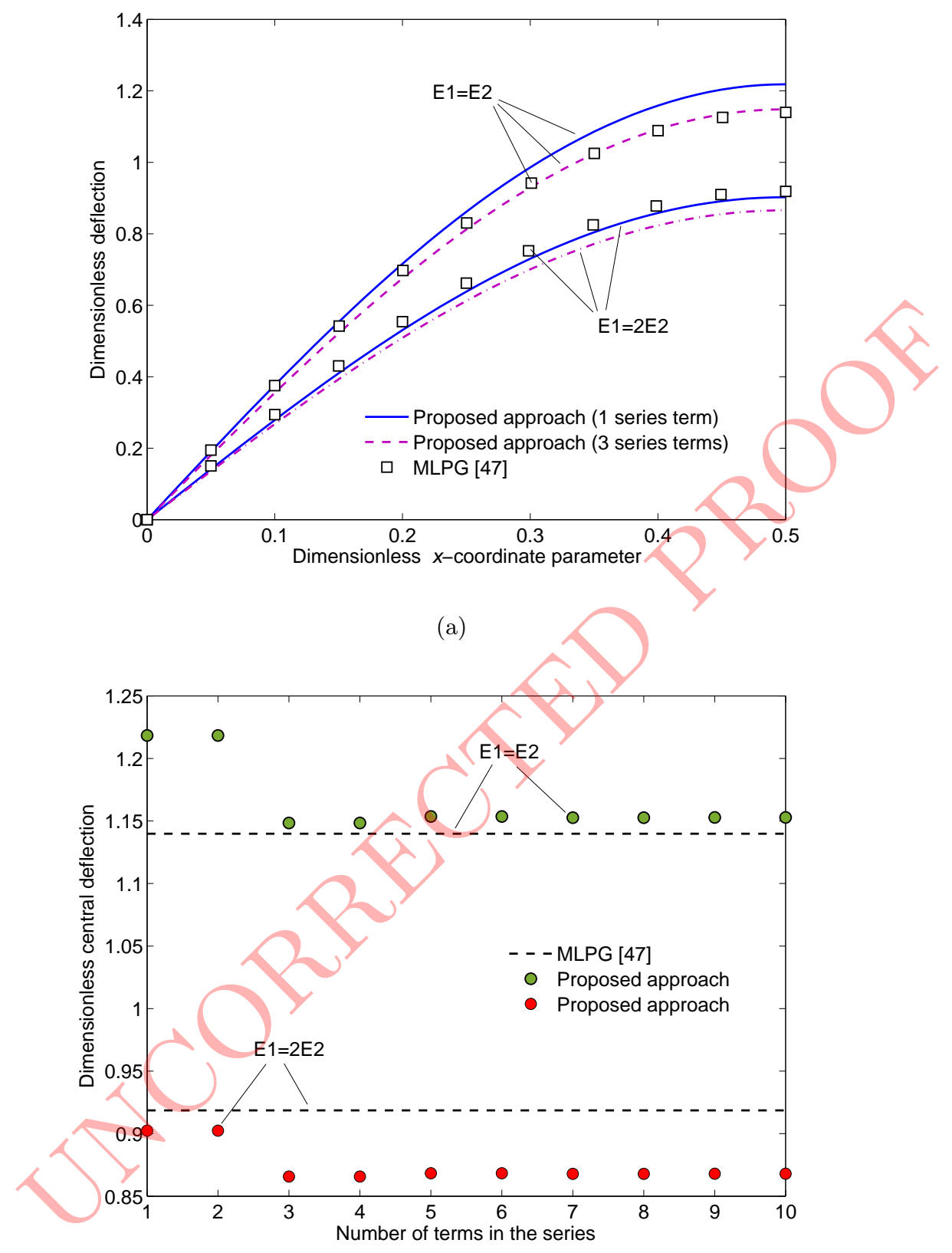

(b)

Figure 5: Comparison of deflection for an orthotropic FG shallow spherical shell with the laterally unrestrained BC subjected to a UDL. Figure (a) shows the variation of dimensionless deflection (i.e. deflection of the shallow shell over deflection of its corresponding flat shell) with the dimensionless $x$-coordinate parameter and Figure (b) demonstrates the convergence of the dimensionless central deflection. 


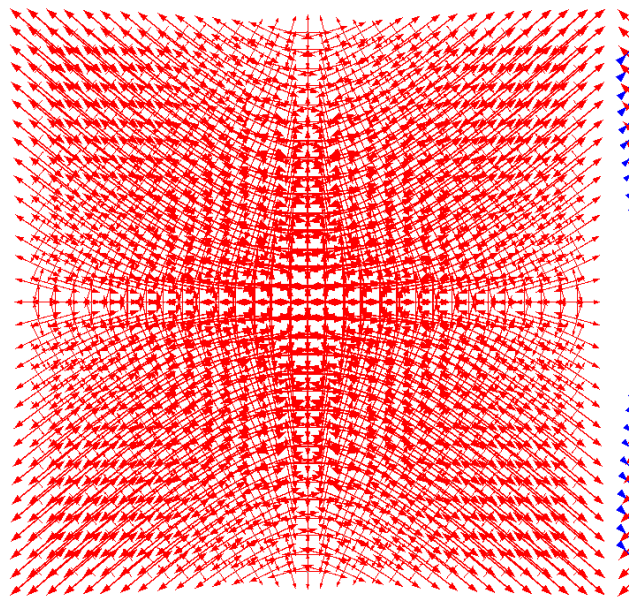

(a) Laterally restrained $\mathrm{BC}$

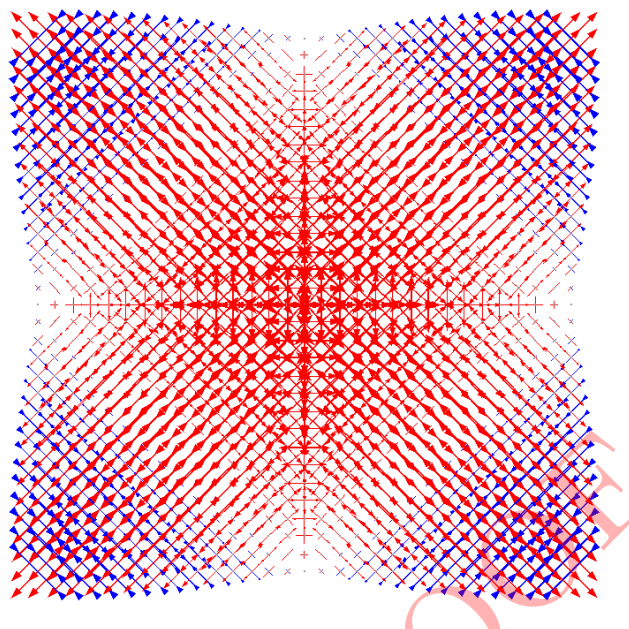

(b) Laterally unrestrained BC

Figure 6: Distribution of all the dimensionless principal membrane tractions in a square orthotropic FG shallow cylindrical shells subjected to a UDL. Blue arrows represent compressive forces and the red ones represent tensile forces.

\subsection{Temperature-dependent shallow shells}

In order to investigate the structural response of shallow shells under fire conditions, a temperature-dependent concrete shallow hyperbolic paraboloidal shell (as the most general case) which is exposed to through-depth thermal gradients resulting from fire conditions has been analysed. Assuming a heat source at the bottom surface of a shell of thickness $0.15 \mathrm{~m}$, two extreme cases of high and low rates of heating are considered [40]:

i. A "short hot" exponential fire representing a high heating rate $(\eta=0.005)$ with a maximum temperature of $1000^{\circ} \mathrm{C}$ when the bottom surface of the shell has been heated for $1,200 \mathrm{~s}$.

ii. A "Long cool" exponential fire representing a low heating rate $(\eta=0.001)$ with a maximum temperature of $650^{\circ} \mathrm{C}$ when the bottom surface of the shell has been heated for $21,600 \mathrm{~s}$.

An FE heat transfer analysis [48, 49] is carried out to determine the temperature history of the imposed heating regimes. As illustrated in Figure 7 , they may 

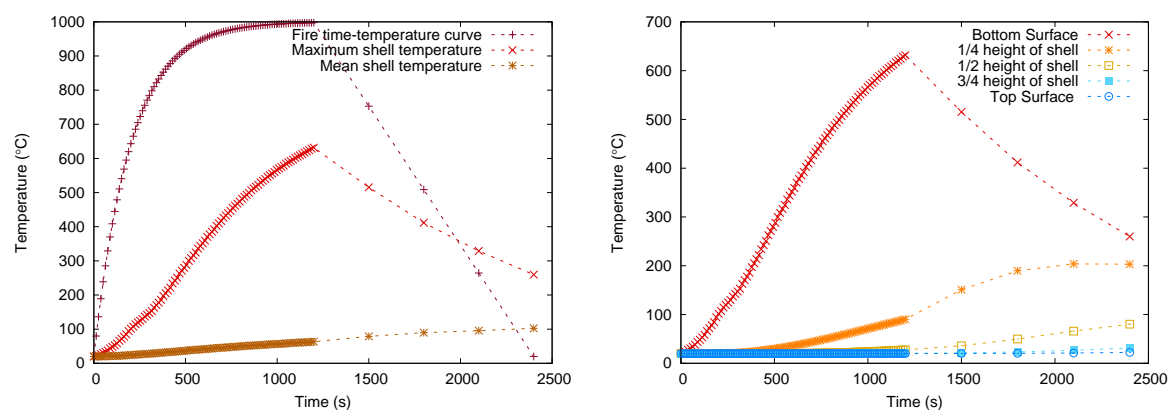

(a) Short hot fire
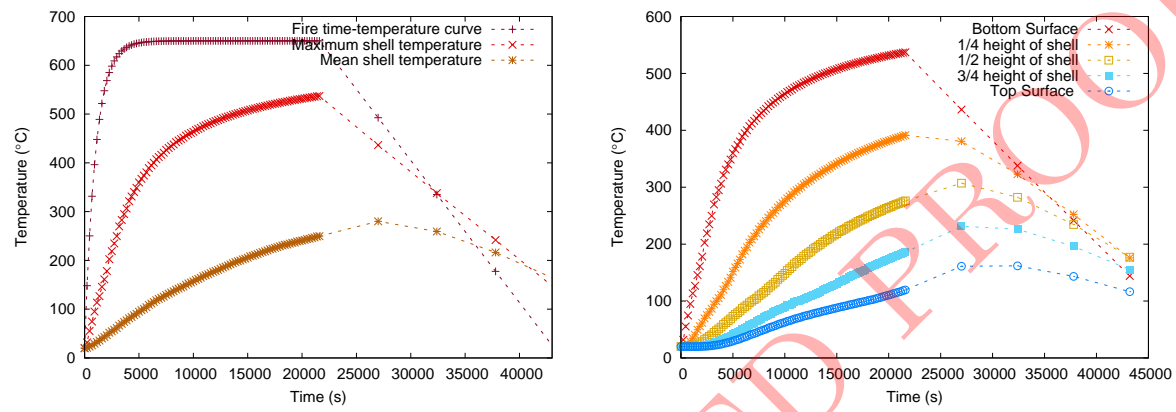

(b) Long cool fire

Figure 7: Time-temperature curves for the two fire scenarios

vary slowly with time or may be sustained over long periods of time. The corresponding through-depth temperature distributions for different times are shown in Figure 8. In this analysis, the curve obtained for $1111 \mathrm{~s}$ is chosen to represent the short hot fire condition and the curve obtained for $3637 \mathrm{~s}$ is chosen to represent the long cool fire condition. Using curve fitting (see Figure 9), the following expressions are obtained for the short hot fire

$$
\theta(z)=20.01+585.30 \exp \left[-9\left(\frac{z}{h}+\frac{1}{2}\right)\right]
$$

and for the long cool fire

$$
\theta(z)=23.39+271.53 \exp \left[-4.5\left(\frac{z}{h}+\frac{1}{2}\right)\right]
$$

The above non-uniform temperature fields can readily be used to study the structural performance of shallow shells under two extreme fire scenarios with 


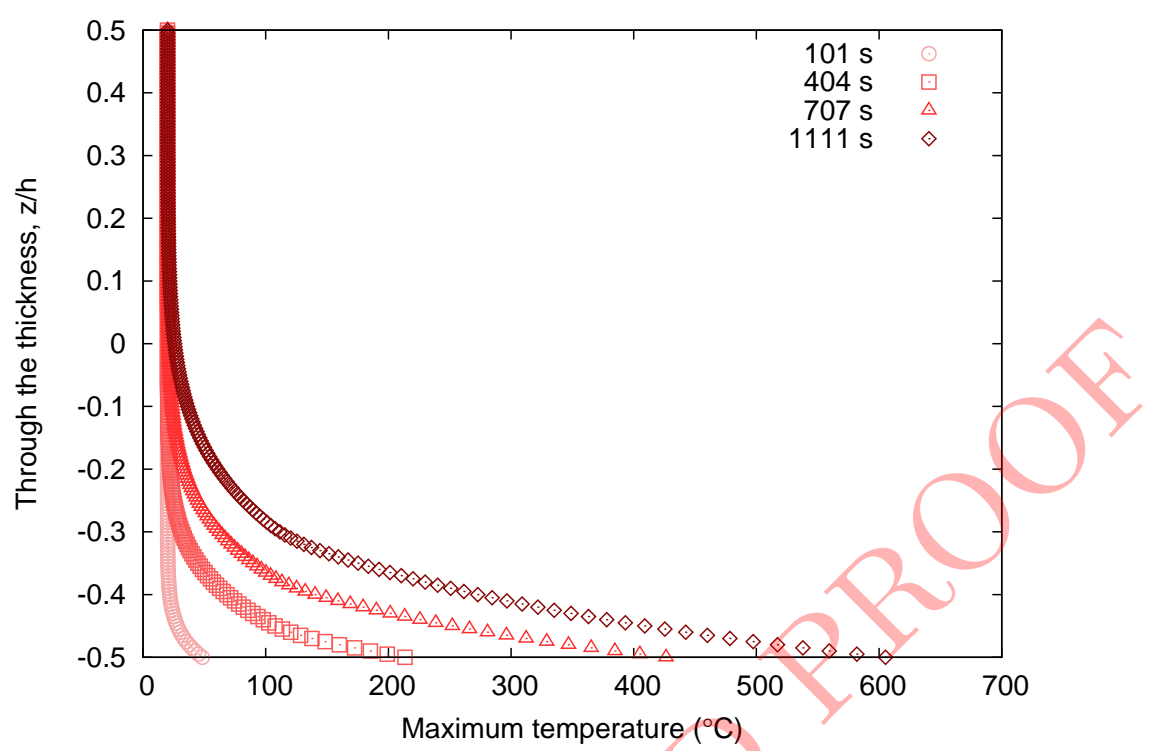

(a) Short hot fire

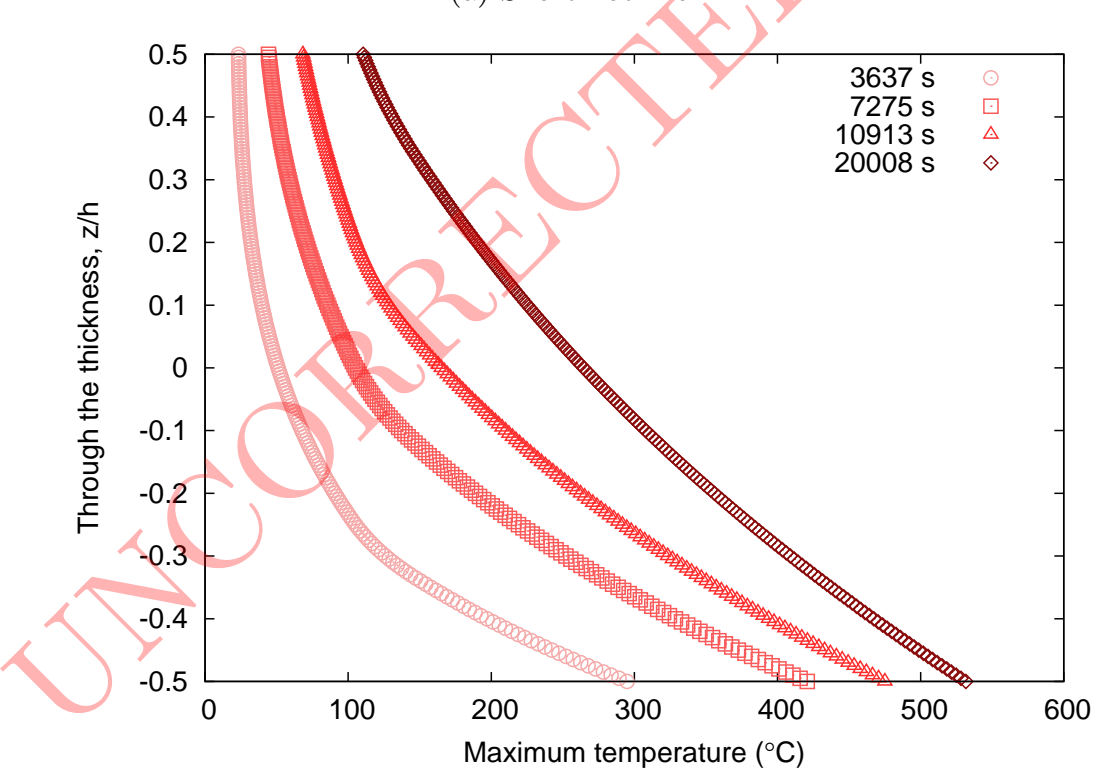

(b) Long cool fire

Figure 8: Through the thickness temperature distributions for the two fire scenarios. 


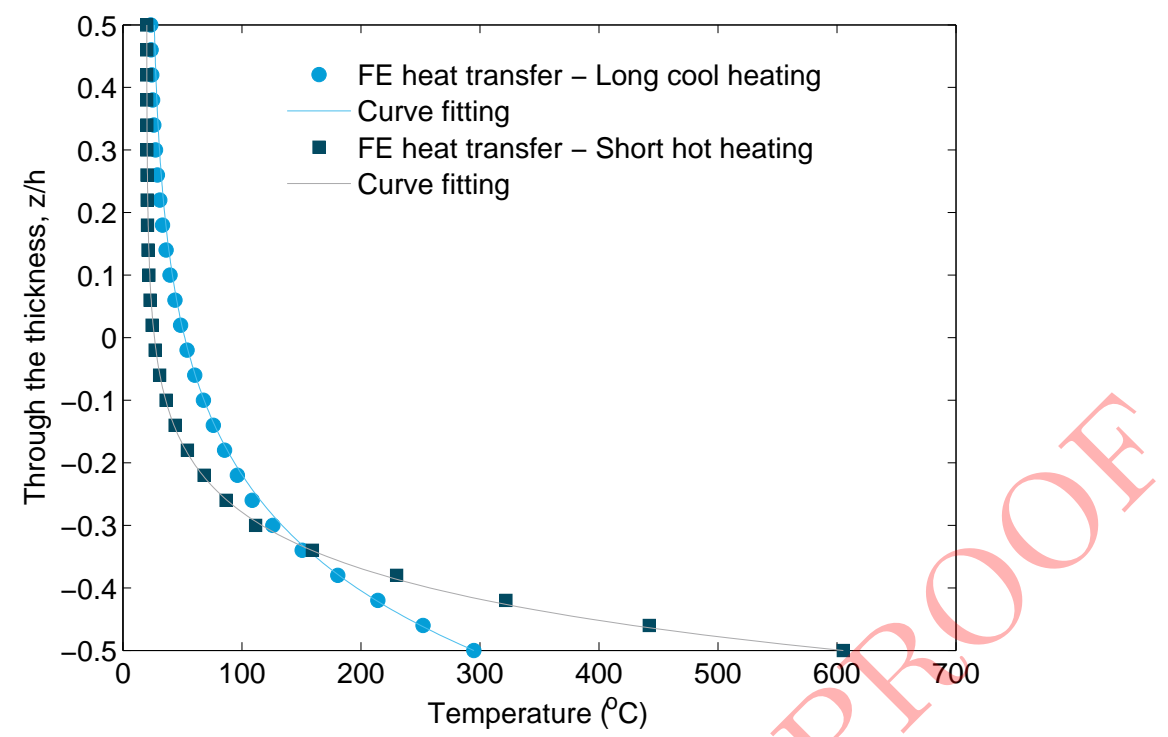

Figure 9: Non-uniform through the thickness temperature distributions for the shell. The assumed curve fitting functions correspond to the high and low rates of heating.

different rates of heating and likely to produce different mechanical responses. For a temperature-dependent concrete shell, the stiffness coefficients are given by

$$
\mathbf{Q}=\left(\begin{array}{ccc}
\frac{E(\theta, z)}{1-\nu^{2}} & \frac{E(\theta, z) \nu}{1-\nu^{2}} & 0 \\
& \frac{E(\theta, z)}{1-\nu^{2}} & 0 \\
\text { symm. } & \frac{E(\theta, z)}{2(1+\nu)}
\end{array}\right)
$$

The following expression which lies between the prediction of both the Eurocode [50] and Australian Standard [51] is assumed for the elastic modulus of concrete [40]

$$
E(\theta, z)=E_{0} \exp \left[-\left(\frac{\theta-52.06}{532}\right)^{2}\right]
$$

where $E_{0}$ is the elastic modulus of the concrete at ambient temperature $\left(27 \times 10^{9}\right.$ $\mathrm{N} / \mathrm{m}^{2}$ ) and $E_{\theta}$ is its counterpart at elevated temperatures. For the coefficient 


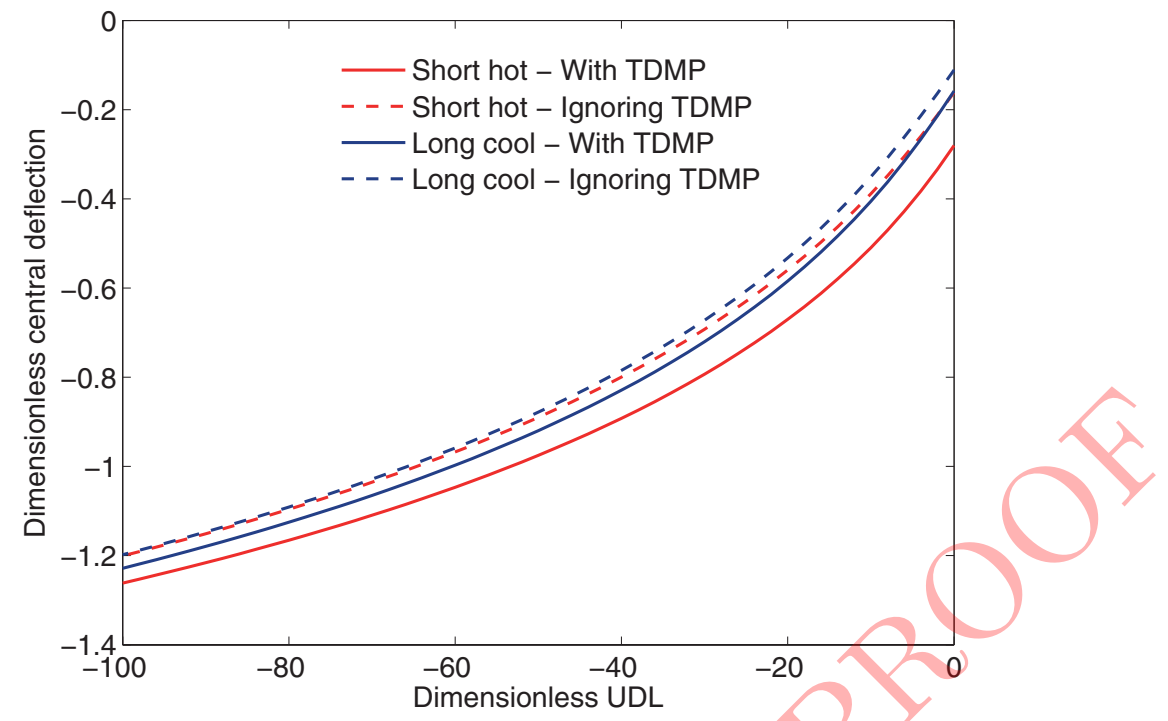

Figure 10: Dimensionless central deflection of square shallow hyperbolic paraboloidal shells ( $h / a=0.03$ and $\left.R_{x} / a=-R_{y} / a=-5\right)$ with the laterally restrained BC subjected to a UDL and the short hot and long cool fire scenarios.

of thermal expansion of the concrete, the following expression is taken [40]

$$
\alpha(\theta, z)= \begin{cases}9 \times 10^{-6}+6.9 \times 10^{-11} \theta^{2}, & 20^{\circ} \mathrm{C} \leq \theta \leq 700{ }^{\circ} \mathrm{C} \\ 0, & 700{ }^{\circ} \mathrm{C}<\theta \leq 1200{ }^{\circ} \mathrm{C}\end{cases}
$$

where $\alpha_{0}$ is the coefficient of thermal expansion of the concrete at ambient temperature $\left(9 \times 10^{-6}\right)$ and $\alpha_{\theta}$ is its corresponding modulus at elevated temperatures. For structural steel, the relevant temperature-dependent material properties (TDMP) can be found in [40].

The load-deflection relationship for a shallow hyperbolic paraboloidal shell is shown in Fígures 10 and 11 for both the chosen BCs. It can be seen that under a downwardly directed UDL, larger deflections are produced in the case of short hot fire. Moreover, the effect of TDMP on the nonlinear response of the shell is more considerable for the short hot fire condition compared with the long cool fire condition. Adding lateral restraint at the shell edges has clearly resulted in lower values for the shell maximum deflection as compared with those values 


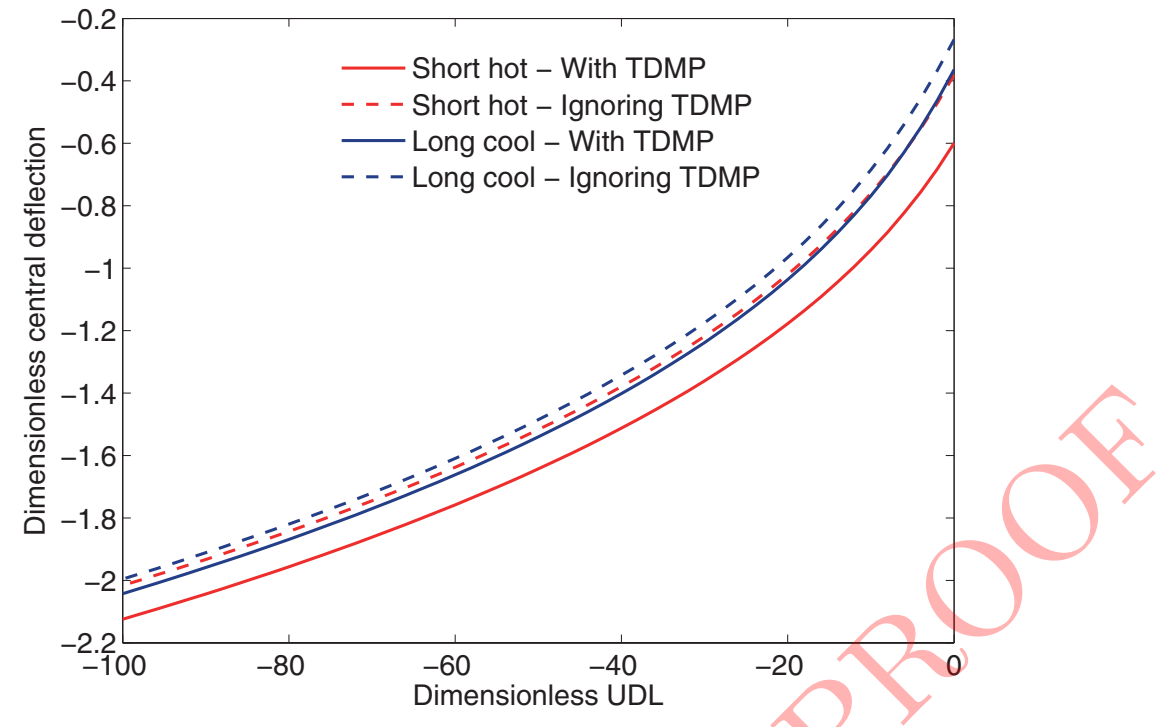

Figure 11: Dimensionless central deflection of square shallow hyperbolic paraboloidal shells ( $h / a=0.03$ and $\left.R_{x} / a=-R_{y} / a=-5\right)$ with the laterally unrestrained BC subjected to a UDL and the short hot and long cool fire scenarios.

obtained for the laterally unrestrained shells.

Quiver plots of all dimensionless principal membrane tractions (forces) of the same shallow hyperbolic paraboloidal shell under the short hot fire exposure condition are illustrated in Figure 12 for both the chosen BCs. Figure 12a shows the compressive forces dominating the shell as a result of lateral restraint along the shell boundaries, producing maximum tensile forces around the shell corners. By moving away from the corners, the forces become more uniform. However, as Figure $12 \mathrm{~b}$ depicts, in the case of laterally unrestrained BC, compressive forces develop along the two shell edges with positive curvature, whereas at the centre of the shell only tensile forces are formed. The highest value of compressive forces occurs in the shell corners, and the overall pattern suggests a clear development of a compressive ring, supporting the tension in the middle. 


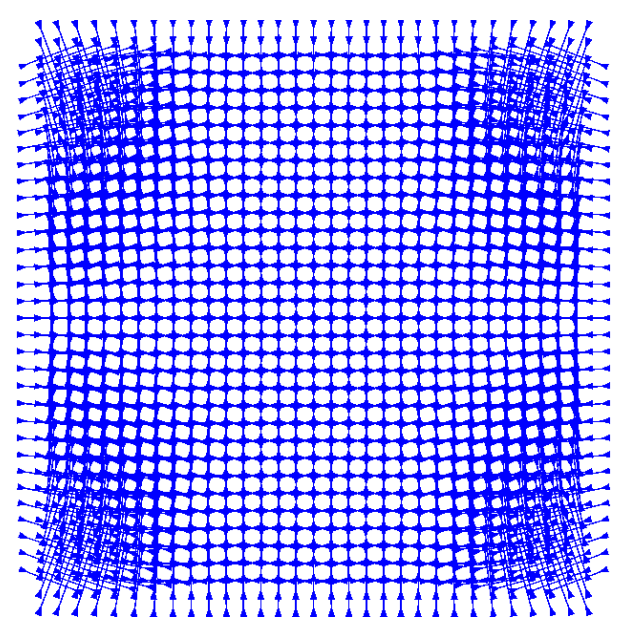

(a) Laterally restrained $\mathrm{BC}$

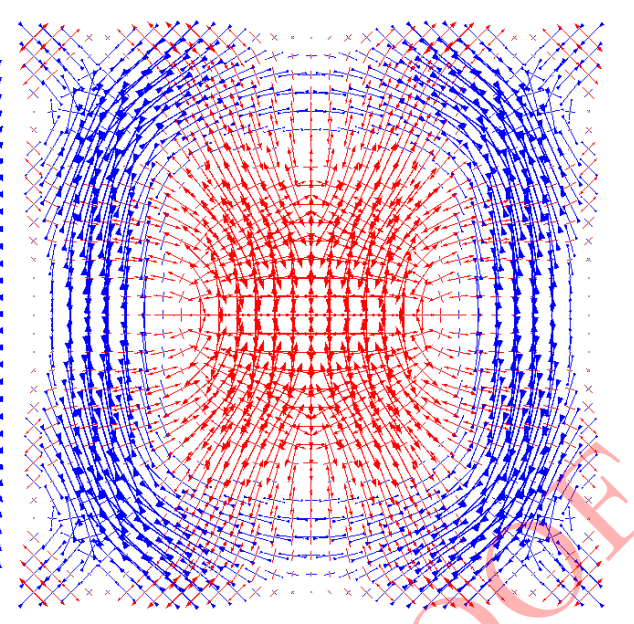

(b) Laterally unrestrained BC

Figure 12: Distribution of the all dimensionless principal membrane tractions in a square temperature-dependent concrete shallow hyperbolic paraboloidal shell under a UDL and the short hot fire exposure condition. Blue arrows represent compressive forces and the red ones represent tensile forces.

\section{Conclusions}

A geometrically and materially nonlinear analysis of temperature-dependent shallow shells subjected to thermo-mechanical loadings was presented. In all cases considered, transverse deflections and rotations along the shell boundaries were assumed to be restrained and free, respectively, while translations across the boundaries could be free or restrained. Based on the analysis presented herein, the following conclusions can be drawn:

- The formulation exhibits very good performance and convergence rate for shallow shells with the laterally restrained BC. For this BC, three series terms were used to achieve an accurate final solution. However, in the case of laterally unrestrained $\mathrm{BC}$, the good performance of the proposed formulation was limited to the case of extremely shallow shells. It is hoped that with further investigation, an accurate method for such shells can be developed. 
- The solutions presented for the laterally restrained BCs, can especially be used for benchmarking FE codes developed for thermo-mechanical simulations of shell elements. Such use is also reasonable for the solutions presented for extremely shallow shells with laterally unrestrained BCs.

- The effect of TDMP on the nonlinear response of the shell is more pronounced when short hot fire exposure condition is imposed. This effect is, however, negligible for the long cool fire exposure conditions.

- The formulation developed is versatile and appropriate for other applications, such as laminated composite or orthotropic FG shallow shells. It can be used for the nonlinear analysis of extremely shallow shells when subjected to non-uniform through-depth thermal gradients, while also considering nonlinear and temperature-dependent material behaviour.

- The stress trajectories and principal stress distribution patterns obtained from the proposed approach were found to be very similar to the ones obtained from the FE analysis. These patterns can be explored and exploited to visualise internal structures in composite flat and curved shells in order to develop much deeper insights into the load-carrying mechanisms of shells.

\section{Acknowledgement}

The authors gratefully acknowledge funding support from the Edinburgh Research Partnership in Engineering (ERPE).

\section{References}

[1] Surana, K., Orth, N.. p-version hierarchical three dimensional curved shell element for heat conduction. Computational Mechanics 1991;7(5-6):341353. 
[2] Bose, A., Surana, K.. Piecewise hierarchical p-version axisymmetric shell element for non-linear heat conduction in laminated composites. Computers \& structures 1993;47(1):1-18.

[3] Ling, C.S., Surana, K.. p-version least squares finite element formulation for axisymmetric heat conduction with temperature-dependent thermal conductivities. Computers \& structures 1994;52(2):353-364.

[4] Djoudi, M., Bahai, H.. A shallow shell finite element for the linear and nonlinear analysis of cylindrical shells. Engineering structures 2003;25(6):769 778.

[5] Talamona, D., Franssen, J.M.. A quadrangular shell finite element for concrete and steel structures subjected to fire. Journal of Fire Protection Engineering 2005;15(4):237-264.

[6] Jeffers, A.E., Beata, P.A.. Generalized shell heat transfer element for modeling the thermal response of non-uniformly heated structures. Finite Elements in Analysis and Design 2014;83:58-67.

[7] Jeffers, A.E.. Triangular shell heat transfer element for the thermal analysis of nonuniformly heated structures. Journal of Structural Engineering $2015 ;: 04015084$.

[8] Kumar, W.P., Palaninathan, R.. Nonlinear response of laminated cylindrical panels. Thin-walled structures 2001;39(6):519-533.

[9] Wang, X. Nonlinear stability analysis of thin doubly curved orthotropic shallow shells by the differential quadrature method. Computer methods in applied mechanics and engineering 2007;196(17):2242-2251.

[10] Panda, S., Singh, B.. Thermal post-buckling behaviour of laminated composite cylindrical/hyperboloid shallow shell panel using nonlinear finite element method. Composite Structures 2009;91(3):366-374. 
[11] Altekin, M., Yukseler, R.F.. Axisymmetric large deflection analysis of fully and partially loaded shallow spherical shells. Structural Engineering and Mechanics 2013;47(4):559-573.

[12] Civalek, Ö.. Geometrically nonlinear dynamic and static analysis of shallow spherical shell resting on two-parameters elastic foundations. International Journal of Pressure Vessels and Piping 2014;113:1-9.

[13] Liew, K., Lim, C., Kitipornchai, S.. Vibration of shallow shells: a review with bibliography. Applied Mechanics Reviews 1997;50(8):431-444

[14] Ganapathi, M.. Dynamic stability characteristics of functionally graded materials shallow spherical shells. Composite structures 2007;79(3):338343.

[15] Shariyat, M.. Dynamic thermal buckling of suddenly heated temperaturedependent fgm cylindrical shells, under combined axial compression and external pressure. International Journal of Solids and Structures 2008;45(9):2598-2612.

[16] Amabili, M.. Nonlinear vibrations and stability of shells and plates. Cambridge University Press; 2008.

[17] Qatu, M.S., Sullivan, R.W., Wang, W.. Recent research advances on the dynamic analysis of composite shells: 2000-2009. Composite Structures 2010;93(1):14-31.

[18] Duc, ২N.D., Quan, T.Q.. Transient responses of functionally graded double curved shallow shells with temperature-dependent material properties in thermal environment. European Journal of Mechanics-A/Solids 2014;47:101-123.

[19] Tornabene, F., Fantuzzi, N., Bacciocchi, M.. Free vibrations of free-form doubly-curved shells made of functionally graded materials using higherorder equivalent single layer theories. Composites: Part B 2014;67:490-509. 
[20] Fantuzzi, N., Tornabene, F., Viola, E.. Four-parameter functionally graded cracked plates of arbitrary shape: A gdqfem solution for free vibrations. Mechanics of Advanced Materials and Structures 2015;23(1):89-107.

[21] Woo, J., Meguid, S.. Nonlinear analysis of functionally graded plates and shallow shells. International Journal of Solids and Structures 2001;38(42):7409-7421.

[22] van Campen, D., Bouwman, V., Zhang, G., Zhang, J., ter Weeme, B.. Semi-analytical stability analysis of doubly-curved orthotropic shallow panelsconsidering the effects of boundary conditions. International journal of non-linear mechanics 2002;37(4):659-667.

[23] Nie, G.. Analysis of non-linear behaviour of imperfect shallow spherical shells on pasternak foundation by the asymptotic iteration method. International journal of pressure vessels and piping 2003;80(4):229-235.

[24] Heuer, R., Ziegler, F.. Thermoelastic stability of layered shallow shells. International Journal of Solids and Structures 2004;41(8):2111-2120.

[25] Duc, N.D., Van Tung, H.. Nonlinear response of pressure-loaded functionally graded cylindrical panels with temperature effects. Composite Structures 2010;92(7):1664-1672.

[26] Shen, H.S.. Postbuckling analysis of axially-loaded functionally graded cylindrical shells in thermal environments. Composites Science and Technology 2002;62(7):977-987.

[27] Shen, H.S.. Postbuckling analysis of axially loaded functionally graded cylindrical panels in thermal environments. International Journal of Solids and Structures 2002;39(24):5991-6010.

[28] Shen, H.S., Leung, A.Y.. Postbuckling of pressure-loaded functionally graded cylindrical panels in thermal environments. Journal of engineering mechanics 2003;129(4):414-425. 
[29] Shen, H.S.. Thermal postbuckling behavior of functionally graded cylindrical shells with temperature-dependent properties. International Journal of Solids and Structures 2004;41(7):1961-1974.

[30] Yang, J., Liew, K., Wu, Y., Kitipornchai, S.. Thermo-mechanical postbuckling of fgm cylindrical panels with temperature-dependent properties. International Journal of Solids and Structures 2006;43(2):307-324.

[31] Shahsiah, R., Eslami, M., Naj, R.. Thermal instability of functionally graded shallow spherical shell. Journal of Thermal Stresses 2006;29(8):771790.

[32] Girish, J., Ramachandra, L.. Thermomechanical postbuckling analysis of cross-ply laminated cylindrical shell panels. Journal of engineering mechanics 2006;132(2):133-140.

[33] Nie, G., Chan, C., Yao, J., He, X.. Asymptotic solution for nonlinear buckling of orthotropic shells on elastic foundation. AIAA journal 2009;47(7):1772-1783.

[34] Amabili, M.. Non-linear vibrations of doubly curved shallow shells. International Journal of Non-Linear Mechanics 2005;40(5):683-710.

[35] Hamed, E., Bradford, M.A., Gilbert, R.I., Chang, Z.T.. Analytical model and experimental study of failure behavior of thin-walled shallow concrete domes. Journal of Structural Engineering 2010;137(1):88-99.

[36] Bich, D.H., Van Tung, H.. Non-linear axisymmetric response of functionally graded shallow spherical shells under uniform external pressure including temperature effects. International Journal of Non-Linear Mechanics 2011;46(9):1195-1204.

[37] Sofiyev, A., Kuruoglu, N.. Buckling analysis of nonhomogeneous orthotropic thin-walled truncated conical shells in large deformation. ThinWalled Structures 2013;62:131-141. 
[38] Xue, J., Yuan, D., Han, F., Liu, R.. An extension of karman-donnell's theory for non-shallow, long cylindrical shells undergoing large deflection. European Journal of Mechanics-A/Solids 2013;37:329-335.

[39] Tornabene, F., Fantuzzi, N., Viola, E., Batra, R.C.. Stress and strain recovery for functionally graded free-form and doubly-curved sandwich shells using higher-order equivalent single layer theory. Composite Structures 2015;119:67-89.

[40] Khazaeinejad, P., Usmani, A., Laghrouche, O.. Temperature-dependent nonlinear behaviour of thin rectangular plates exposed to through-depth thermal gradients. Composite Structures 2015;132:652-664.

[41] Drysdale, D.. An introduction to fire dynamics. John Wiley \& Sons; 2011.

[42] Donnell, L.H.. Beams, plates and shells. McGraw-Hill New York; 1976.

[43] Qatu, M.S.. Vibration of laminated shells and plates. Elsevier; 2004.

[44] Narimani, R., Khorramabadi, M.K., Khazaeinejad, P.. Mechanical buckling of functionally graded cylindrical shells based on the first order shear deformation theory. In: ASME 2007 Pressure Vessels and Piping Conference. American Society of Mechanical Engineers; 2007, p. 399-404.

[45] Khorramabadi, M.K., Khazaeinejad, P.. On stability of stiffened cylindrical shells with varying material properties. World Academy of Science, Engineering and Technology 2009;56:248-252.

[46] Khazaeinejad, P., Najafizadeh, M.. Mechanical buckling of cylindrical shells with varying material properties. Proceedings of the Institution of Mechanical Engineers, Part C: Journal of Mechanical Engineering Science 2010;224(8):1551-1557.

[47] Sladek, J., Sladek, V., Zhang, C., Solek, P.. Static and dynamic analysis of shallow shells with functionally graded and orthotropic material properties. Mechanics of Advanced Materials and Structures 2008;15(2):142-156. 
[48] Huang, H.C., Usmani, A.S.. Finite element analysis for heat transfer. Springer; 1994.

[49] Usmani, A., Chung, Y., Torero, J.L.. How did the WTC towers collapse: A new theory. Fire Safety Journal 2003;38(6):501-533.

[50] Eurocode, . EN 1993-1-2, Eurocode 3: Design of Steel Structures, Part 1-2: General rules - Structural fire design. 2005.

[51] Standards Australia,. Concrete structures, AS3600. 2001. 